\title{
Generation of a buoyancy-driven coastal current by an Antarctic Polynya
}

Article

Published Version

Wilchinsky, A. V. and Feltham, D. L. (2008) Generation of a buoyancy-driven coastal current by an Antarctic Polynya. Journal of Physical Oceanography, 38 (5). pp. 1011-1032. ISSN 1520-0485 doi: https://doi.org/10.1175/2007JPO3831.1 Available at https://centaur.reading.ac.uk/34719/

It is advisable to refer to the publisher's version if you intend to cite from the work. See Guidance on citing.

Published version at: $\mathrm{http}: / / \mathrm{dx}$.doi.org/10.1175/2007JP03831.1

To link to this article DOI: http://dx.doi.org/10.1175/2007JPO3831.1

Publisher: American Meteorological Society

All outputs in CentAUR are protected by Intellectual Property Rights law, including copyright law. Copyright and IPR is retained by the creators or other copyright holders. Terms and conditions for use of this material are defined in the End User Agreement.

\section{www.reading.ac.uk/centaur}

\section{CentAUR}

Central Archive at the University of Reading

Reading's research outputs online 


\title{
Generation of a Buoyancy-Driven Coastal Current by an Antarctic Polynya
}

\author{
AleXander V. Wilchinsky \\ Centre for Polar Observation and Modelling, Department of Earth Sciences, University College London, London, United Kingdom \\ Daniel L. Feltham \\ Centre for Polar Observation and Modelling, Department of Earth Sciences, University College London, London, and British \\ Antarctic Survey, Cambridge, United Kingdom
}

(Manuscript received 25 April 2007, in final form 9 October 2007)

\begin{abstract}
Descent and spreading of high salinity water generated by salt rejection during sea ice formation in an Antarctic coastal polynya is studied using a hydrostatic, primitive equation three-dimensional ocean model called the Proudman Oceanographic Laboratory Coastal Ocean Modeling System (POLCOMS). The shape of the polynya is assumed to be a rectangle $100 \mathrm{~km}$ long and $30 \mathrm{~km}$ wide, and the salinity flux into the polynya at its surface is constant. The model has been run at high horizontal spatial resolution (500 $\mathrm{m}$ ), and numerical simulations reveal a buoyancy-driven coastal current. The coastal current is a robust feature and appears in a range of simulations designed to investigate the influence of a sloping bottom, variable bottom drag, variable vertical turbulent diffusivities, higher salinity flux, and an offshore position of the polynya. It is shown that bottom drag is the main factor determining the current width. This coastal current has not been produced with other numerical models of polynyas, which may be because these models were run at coarser resolutions. The coastal current becomes unstable upstream of its front when the polynya is adjacent to the coast. When the polynya is situated offshore, an unstable current is produced from its outset owing to the capture of cyclonic eddies. The effect of a coastal protrusion and a canyon on the current motion is investigated. In particular, due to the convex shape of the coastal protrusion, the current sheds a dipolar eddy.
\end{abstract}

\section{Introduction}

Coastal gravity currents are observed frequently in the ocean. A volume of buoyant or relatively dense water tends to spread under the action of gravity at the surface or on the bottom, respectively. When there are no constraints on the flow, the Coriolis force will turn the flow until a motion satisfying a geostrophic balance is achieved. However, when a coast is present, the along-coast pressure gradient force is not balanced by the Coriolis force so that the fluid will flow along the coast, generating a coastal current. The most common origin of buoyant coastal currents is the freshwater discharge from rivers into oceans. Other examples are the East Greenland Current (Wadhams et al. 1979; Bacon et al. 2002), driven by a complex system of forces re-

Corresponding author address: Alexander Wilchinsky, CPOM, Dept. of Earth Sciences, University College London, Gower St., London, WC1E 6BT, United Kingdom.

E-mail: aw@cpom.ucl.ac.uk sulting from the inflow of low salinity water from Fram Strait, meltwater runoff from the Greenland ice sheet, and a predominantly cyclonic wind stress; the Norwegian Coastal Current caused by the freshwater outflow and runoff from the Baltic and Norway, respectively (Johannessen and Mork 1979); and the Leeuwin Current carrying warm water from low latitudes poleward along the west coast of Australia.

In contrast to surface buoyant currents, bottom gravity currents are more difficult to observe owing to their inaccessibility and intermittent character. Cooling, evaporation, or salinization by surface freezing followed by descent of the higher-density water onto the ocean bottom can lead to bottom gravity current formation (Griffiths 1986; Ivanov et al. 2004). These currents may then descend over continental shelves and participate in deep-water formation. The most wellknown examples of this occur in Denmark Strait and at the Mediterranean outflow into the Atlantic. When relatively dense water forms near a vertical boundary under rotation, laboratory experiments reveal two 
mechanisms of mass transport: eddies formed around the density source and a Coriolis-driven boundary current with the boundary to the left (right) of the current in the Southern (Northern) Hemisphere (Condie and Ivey 1988; Davies et al. 1991; Davies and Ahmed 1996).

The densest varieties of deep water associated with high southern latitudes are produced in the many coastal polynyas surrounding Antarctica where continual sea ice formation and export results in brine release into the upper layer of the ocean (Baines and Condie 1998). Antarctic polynyas can be formed at a coast or next to landfast ice (Morales Maqueda et al. 2004). On the time scale of a month relevant to dense water descent in a polynya, the landfast ice can be considered as effectively stationary, and here we refer to both land and landfast ice edges as a coast. Thus, we will focus on coastal polynyas; however, we will also consider several cases where polynyas are formed up to $15 \mathrm{~km}$ offshore, which may occur when landfast sea ice is present.

The first model in which a localized buoyancy forcing was used to describe the effect of an Antarctic polynya on the ocean was proposed by Grumbine (1991). The model was similar to those used by Killworth (1985) and Ikeda (1987) to study the thermocline and it did not resolve instabilities. Gawarkiewicz and Chapman (1995) used a three-dimensional primitive equation model (SPEM) to study a coastal polynya in the Arctic with the vertical flow structure interpolated by nine Chebyshev polynomials. The presence of the polynya was modeled by imposing a temporally constant density flux over a semielliptic ocean surface area adjacent to the coast. The model resolution was $1.03 \mathrm{~km}$ in space and $288 \mathrm{~s}$ in time. Their main result was that. after an initial period of geostrophic adjustment, the presence of the coast and bottom friction caused anomalous flow near the polynya edges that served as a trigger for baroclinic destabilization of the flow and generation of eddies. The eddies then moved offshore, across isobaths, with visible along-slope deviation caused by the Coriolis force only for steep bottom slopes (0.005). Importantly, no coastal current was produced by the numerical model in contrast to laboratory experiments (Condie and Ivey 1988; Davies et al. 1991; Davies and Ahmed 1996), although a short, 15-km-long coastal bottom salinity protuberance developed near the end of their run. Kukichi et al. (1999) modeled offshore transport of dense water generated by a polynya infinitely long in the alongshore direction, but the lack of alongshore variation prevented investigation of coastal currents. Three-dimensional simulations of idealized Denmark Strait outflows by Jiang and Garwood (1996) and Ezer (2005) using different models with different grid sizes did not show any distinct coastal current. However, in these cases due to the higher density difference $\left(0.3 \mathrm{~kg} \mathrm{~m}^{-3}\right)$, higher bottom slope (0.01), and imposed dense water influx, the flows had a much larger alongslope component and the main body of the plume attached itself to the coast. The grid resolution and vertical viscosity play a significant role in directing the current offshore (Legg et al. 2006). Jiang and Garwood (1996) note that within the main body of the plume there is a current that flows along an isobath, but this current does not protrude from the main plume. Gawarkiewicz (2000) applied the original polynya model of Gawarkiewicz and Chapman (1995) to study the dense water offshore transport across a continental shelf break. His calculations showed that, when the dense water reaches the shelf break, an along-slope current is formed. This current is stable and is driven by the same mechanism as a coastal current would be, although no coastal current was captured by the model.

The aim of this work is to demonstrate that negative buoyancy production inside an Antarctic polynya can lead to generation of a coastal current over a continental shelf and to investigate those factors affecting the coastal current intensity and width. The remainder of this paper is structured as follows: section 2 describes our ocean model and domain configuration; section 3 describes our basic simulation and presents a discussion of instability mechanisms leading to eddies in the main plume and coastal current; section 4 describes the effect of different model configurations (bottom slope, salinity flux, bottom drag, and polynya position); section 5 discusses the model simulations in more detail; section 6 describes the effect of coastal and bottom topography on the coastal current; and, finally, in section 7 we summarize our main results and present some concluding remarks.

\section{Model setup}

We use a hydrostatic, three-dimensional primitive equation ocean model initially developed for the simulation of shelf seas, known as the Proudman Oceanographic Laboratory Coastal Ocean Modeling System (POLCOMS). A detailed description of the model is given by Holt and James $(1999,2001)$. The model is run on an Arakawa B grid, uses a sigmacoordinate transformation, and the Piecewise Parabolic Method advection scheme (James 1996). Ocean density is calculated using the United Nations Educational, Scientific and Cultural Organization (UNESCO) Equation of State (Mellor 1991), with neglected dependence on pressure. A typical salinity difference between water masses in our numerical experiments is 0.1 practical salinity units (psu). With this salinity difference, the 
horizontal density gradient varies due to its pressure dependence by less than $0.5 \%$ over the $400-\mathrm{m}$ ambient depth adopted in our model, and the pressure dependence is therefore negligible. Similarly, the effect of the pressure dependence on the barotopic component of the pressure gradient force is negligble: in our simulations the ocean surface height typically varies by about $0.05 \mathrm{~m}$ so that the resulting barotropic pressure gradient force is affected by the pressure dependence of the density by less than $0.25 \%$ over the $400-\mathrm{m}$ depth. Because we consider the potential temperature of the ocean in our calculations to be constant, thermobaric effects cannot play any role. The Mellor-YamadaGalperin level-2.5 turbulence closure scheme with an algebraic mixing length is used to estimate the vertical viscosity and eddy diffusivity (Mellor and Yamada 1974; Galperin et al. 1988): There is no horizontal eddy diffusivity, and the horizontal viscosity is similarly taken to be zero. The upper ocean surface is a free surface, and at the ocean bottom a quadratic drag law is used with a drag coefficient taken here to be 0.005 . The model does not include any sidewall drag treatment, and on sidewalls a no-slip condition is applied. As zero horizontal viscosity is assumed, the no-slip condition affects the momentum balance only through the advective terms near the coast. Because the turbulent closure treats unstably stratified water columns inadequately (Deleersnijder and Luyten 1994), a convective adjustment scheme, similar to that used by Gawarkiewicz and Chapman (1995), is employed in POLCOMS: the salinity and temperature are replaced by their mean values in an upper layer whose thickness is the minimum thickness that provides a stable stratification after the adjustment.

To simplify comparison of our results with previous polar offshore transport models, the model is run in a Cartesian coordinate system with the Coriolis parameter being $-1.33 \times 10^{-4}$, determined by a southern latitude of $66^{\circ}$, which is the approximate latitude of the East Antarctic coast. Here, we focus on continental shelf dynamics. A typical Antarctic continental shelf depth is $400 \mathrm{~m}$, with the distance from the coast to the $1-\mathrm{km}$ depth isobath being about $200 \mathrm{~km}$ between $90^{\circ} \mathrm{E}$ and $180^{\circ}$ (Baines and Condie 1998). The basic model geometry is given by a rectangular basin $450 \mathrm{~km}$ long along the $x$ axis, $130 \mathrm{~km}$ wide along the $y$ axis, and 400 $\mathrm{m}$ deep along the $z$ axis (see Fig. 1). The coast is given by $y=1 \mathrm{~km}$ and the coastal boundary is assumed to be vertical with zero salinity and temperature fluxes across it. To avoid any salinity sink by imposing a constant salinity at the open ocean boundaries, we impose zero salinity flux and zero velocity at the open boundaries, effectively treating them as rigid walls. The initial sea

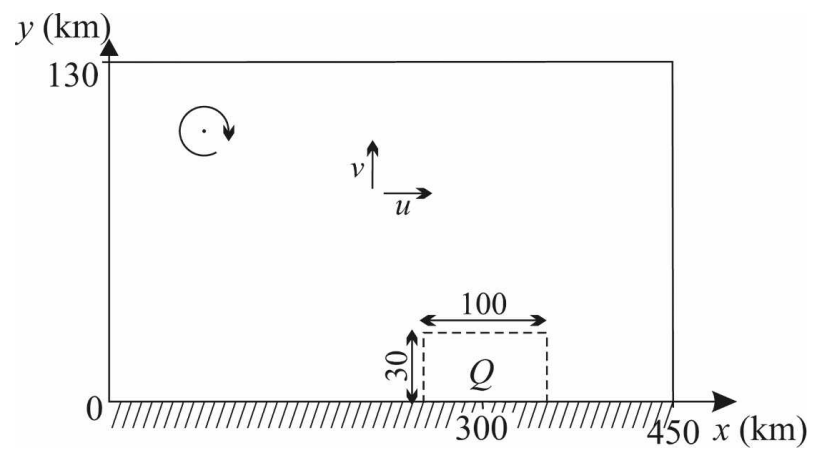

FIG. 1. The basic geometry of the model: plan view.

surface height is taken to be constant. Ocean potential temperature remains uniform throughout the domain at $0^{\circ} \mathrm{C}$ and the initial salinity is uniform at $34.5 \mathrm{psu}$, which are typical values for the upper layer of East Antarctica waters (Rintoul et al. 1997).

Polynya lengths can vary between several hundred meters to hundreds of kilometers depending on location and time of year (Smith et al. 1990), with typical East Antarctica polynya areas in the range from 1000 to $23000 \mathrm{~km}^{2}$ (Massom et al. 1998). Here we study a rectangular polynya $100 \mathrm{~km}$ long and $30 \mathrm{~km}$ wide, which is at the lower end of the area range but quite typical. The rectangular shape of the polynya was chosen so that it can be moved off the coast without breaking its symmetry, which would not be the case for a semielliptic polynya adopted by Gawarkiewicz and Chapman (1995). The salinity flux into the upper surface of the polynya is set to be constant. As POLCOMS assumes zero surface salinity flux, so as not to break the integrity of the code, the polynya salinity flux is realized through addition of the corresponding amount of salt to the uppermost sigma layer at every time step. Because a convective adjustment is used, we do not expect any error to be introduced by employing this technique.

Our polynya and domain size makes it practical to use a high spatial resolution grid of $500 \mathrm{~m}$ in order to capture small-scale processes, although our model results were essentially unaltered at a coarser resolution of $750 \mathrm{~m}$. The vertical resolution is determined by 40 equidistant sigma layers (10-m vertical resolution) with the salinity and velocities given at their centers. We used a barotropic time step of $6 \mathrm{~s}(4 \mathrm{~s}$ in our calculations with a bottom slope of 0.005 ) and a baroclinic time step 50 times larger than this. All runs were terminated when the open ocean boundary conditions began to influence the simulations.

\section{Basic case}

In our basic case, the polynya is adjacent to the coast with its center positioned at $x=300 \mathrm{~km}$ and the ocean 


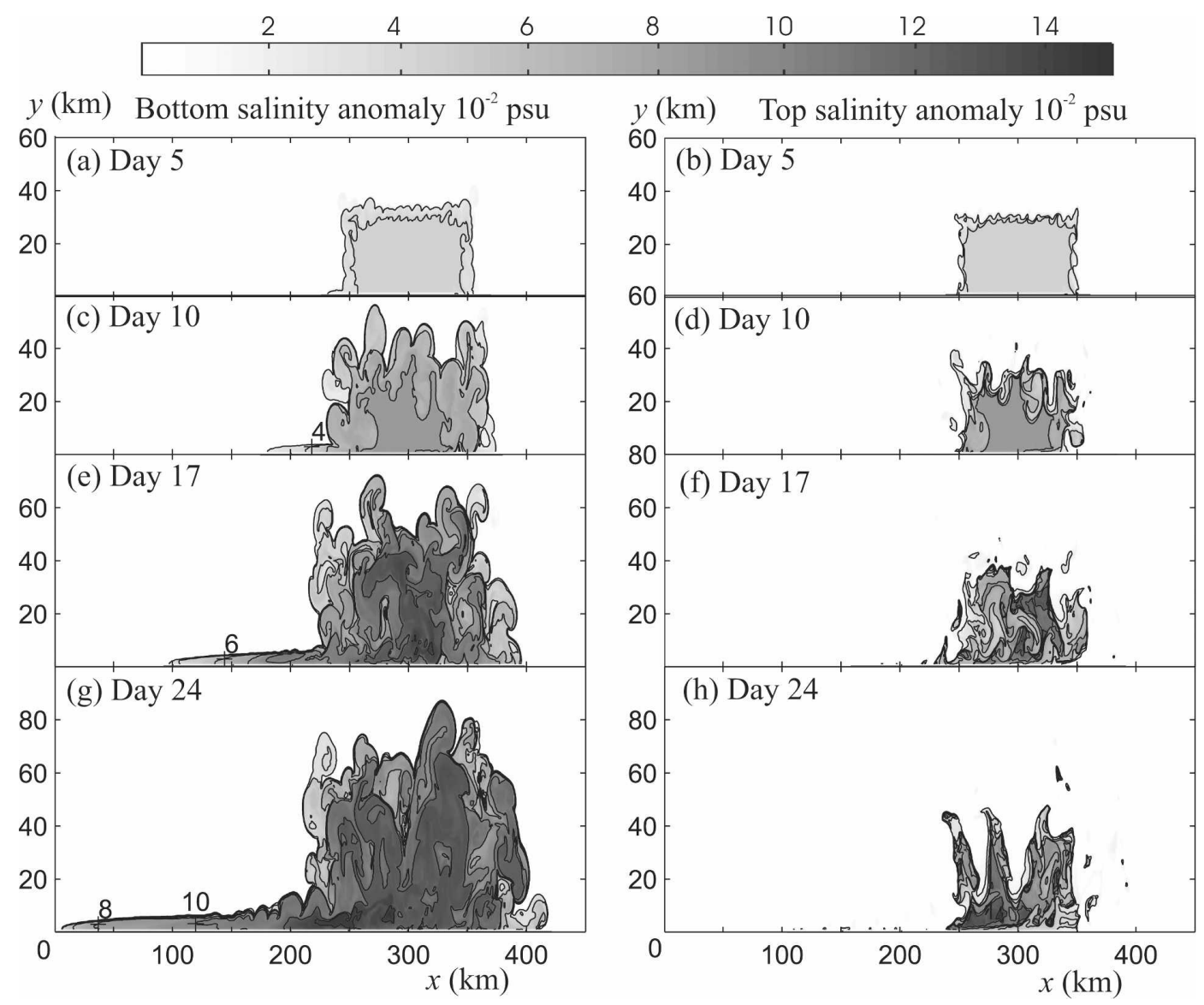

FIG. 2. The salinity anomaly distribution at (left) bottom and (right) top for the basic case. Contour intervals are 0.02 psu with 0.02 as the minimum.

bottom is horizontal (i.e., there is no slope). The salinity flux into the upper surface of the polynya is set to be $Q=-4 \times 10^{-5}$ psu $\mathrm{m} \mathrm{s}^{-1}$, which approximates a threemonth average freezing rate of $0.1 \mathrm{~m} \mathrm{day}^{-1}$ (Cavalieri and Martin 1985). In Fig. 2, we show the temporal evolution of the salinity anomalies at the bottom and top of the water column, using contour intervals of $0.02 \mathrm{psu}$, some of which are marked for clarity. After the initial period, it is seen that the dense fluid flow effectively consists of a main body permeated with eddies that transport the dense water away from the forcing region (which for the sake of convenience we shall refer to as a plume) and a coastal current moving to the west. Until day 5 the vertical salinity distribution is almost homogeneous, which is similar to the results of Gawarkiewicz and Chapman (1995), who described this period as one of geostrophic adjustment during which the edge of their semielliptic polynya was stable. In contrast to Gawarkiewicz and Chapman, our model immediately produces visible frontal instabilities originating at the polynya corners, where the uniformity of the dense ambient water interface is broken down. To ascertain that the corner perturbation is not an effect of the jump in the salinity flux across the polynya edge, we also considered a semielliptic polynya shape with the salinity flux gradually subsiding to zero across a buffer region, exactly as was done by Gawarkiewicz and Chapman. In this case, the instabilities occurred in a similar way but they originated around the outer buffer region edge at the steps in polynya width, which result from the approximation of a semielliptical boundary on a Cartesian grid. The instabilities were initially invisible around the ellipse shortest semiaxis where the ellipse curvature is minimal, and therefore perturbations around the two-dimensional flow solution across the polynya edge are also minimal.

As can be seen from a higher-resolution plot of the velocities and the sea surface height anomaly at day 5 given in Fig. 3, geostrophic adjustment occurs in a nontrivial way. Cyclonic eddies appear due to vortex tube stretching as dense water spreads away from the forcing region at the polynya corners. Geostrophic adjustment 

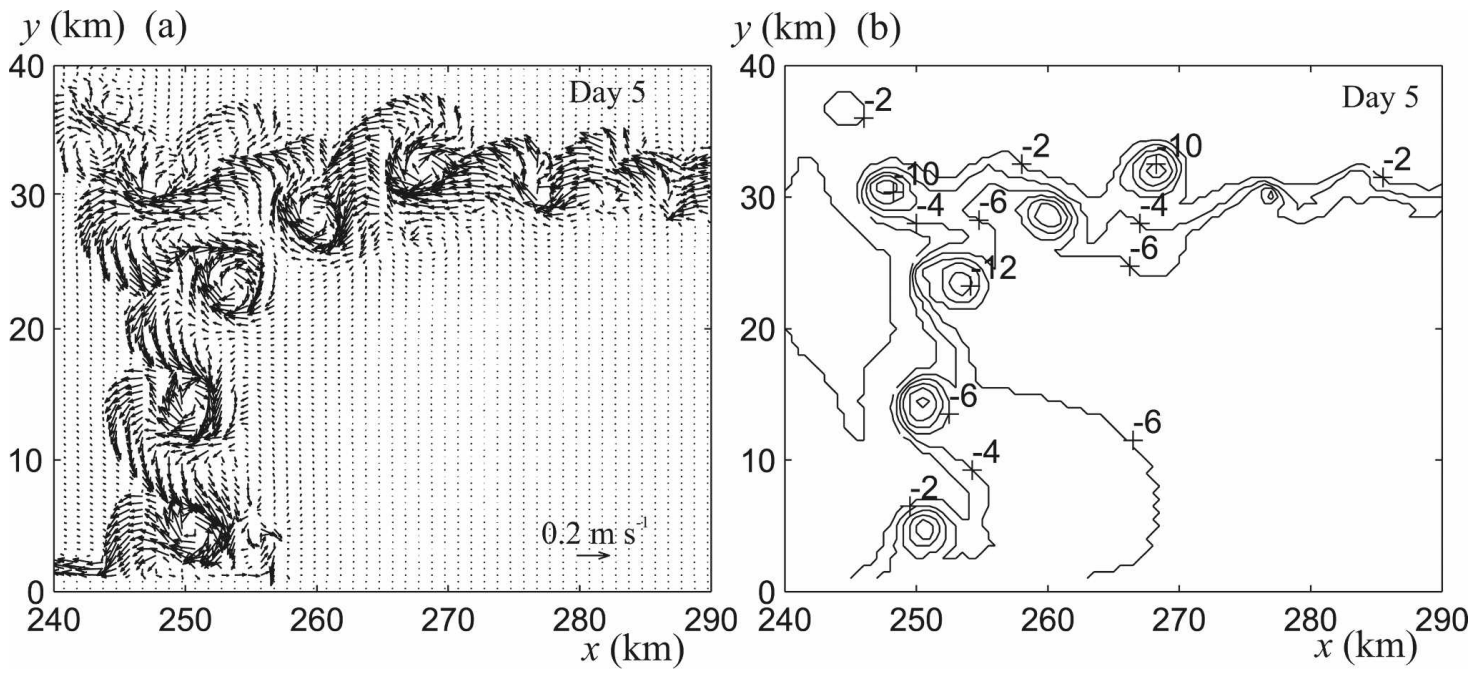

FIG. 3. The (left) velocity and (right) sea surface height (in $10^{-3} \mathrm{~m}$ ) at day 5 for the basic case.

then produces anticyclonic flow that removes some of the fluid moving in the cyclonic eddies in the off-front direction and supplies it to the closest eddy positioned anticlockwise as on-polynya flow. Because the flow in the eddies is circular, the mean flow is determined by this anticyclonic motion, which characterizes geostrophic adjustment. The sea surface height anomalies shown in Fig. $3 b$ are very similar to those of the most baroclinically unstable modes of a gravity current described by Swaters (1991, Fig. 9c therein). The typical distance between the eddies is $\lambda=10 \mathrm{~km}$. We denote the internal Rossby deformation radius as $R=$ $\left(g^{\prime} h\right)^{1 / 2} / f$, where $h$ is the dense water depth (equal to the basin depth $H=400 \mathrm{~m}$ during the initial period), and the reduced gravity is $g^{\prime}=g\left(\rho-\rho_{a}\right) / \rho_{o}$, where $\rho$ and $\rho_{a}$ are the dense and ambient water densities, and $\rho_{o}$ is the density of pure water at $0^{\circ} \mathrm{C}$. The instabilities originate when the salinity anomaly ranges between 0.02 and 0.04 psu, yielding a nondimensional instability wavelength of $\gamma \sim 0.6-0.85$, where

$$
\gamma=\frac{\lambda}{2 \pi R} .
$$

This estimate of $\gamma$ is similar to that obtained by Swaters for the most unstable mode, with a typical value of 0.7 within a range of about 0.4-1.0.

That the eddies become important to mixing across the polynya after day 5 may be understood by noting that by day 5 the Rossby radius of deformation becomes of similar size to the polynya width. When the salinity anomaly (determining $g^{\prime}$ ) reaches about 0.04 psu, the Rossby radius $R=\left(g^{\prime} h\right)^{1 / 2} / f=26 \mathrm{~km}$ approaches the polynya width of $30 \mathrm{~km}$. The salinity anomaly in the eddies as they first form is given by
$Q t / H$ [i.e., the salinity is distributed approximately equally over the water column in the polynya because there is no significant horizontal mixing (Gawarkiewicz and Chapman 1995)], so the time at which the Rossby radius reaches the polynya width may thus be estimated from $Q t / H \approx 0.04$ psu to be about 5 days.

The evolution of the bottom velocities and the surface velocities at the last day of the simulation are shown in Fig. 4. It can be clearly seen that the dynamic disturbances propagate from the polynya edges inward and outward and that by day 17 the whole plume region is involved in vigorous mixing. The mass supply by the advancing plume and coastal current is balanced by an opposite motion of the upper layer; although this is less clear in the eddies constituting the main plume, an eastward surface current along the coast is clearly visible, which might be observed in the motion of sea ice next to the coast. Furthermore, some generated eddies have opposing rotation in their upper and lower parts (e.g., at $x=325 \mathrm{~km}, y=80 \mathrm{~km}$ on day 24). This behavior is similar to that of the steady-state geostrophic adjustment solution with the upper and lower layers rotating in opposite directions (Dewar and Killworth 1990). From the bottom salinity distribution (Fig. 2), and especially from the salinity distribution along the coast $(y=1 \mathrm{~km})$ presented in Fig. 5, it is clear that the left-hand side of the plume is denser than the righthand side; this is caused by the transport of dense water by the coastal current to the left of the plume. Vertical mixing upstream disrupts the monotonic salinity distribution characteristic of the frontal region.

Inspection of Fig. 2 shows that eddies appear in the coastal current. To visualize them more clearly, in Fig. 6 we plot at higher resolution model output for the part 

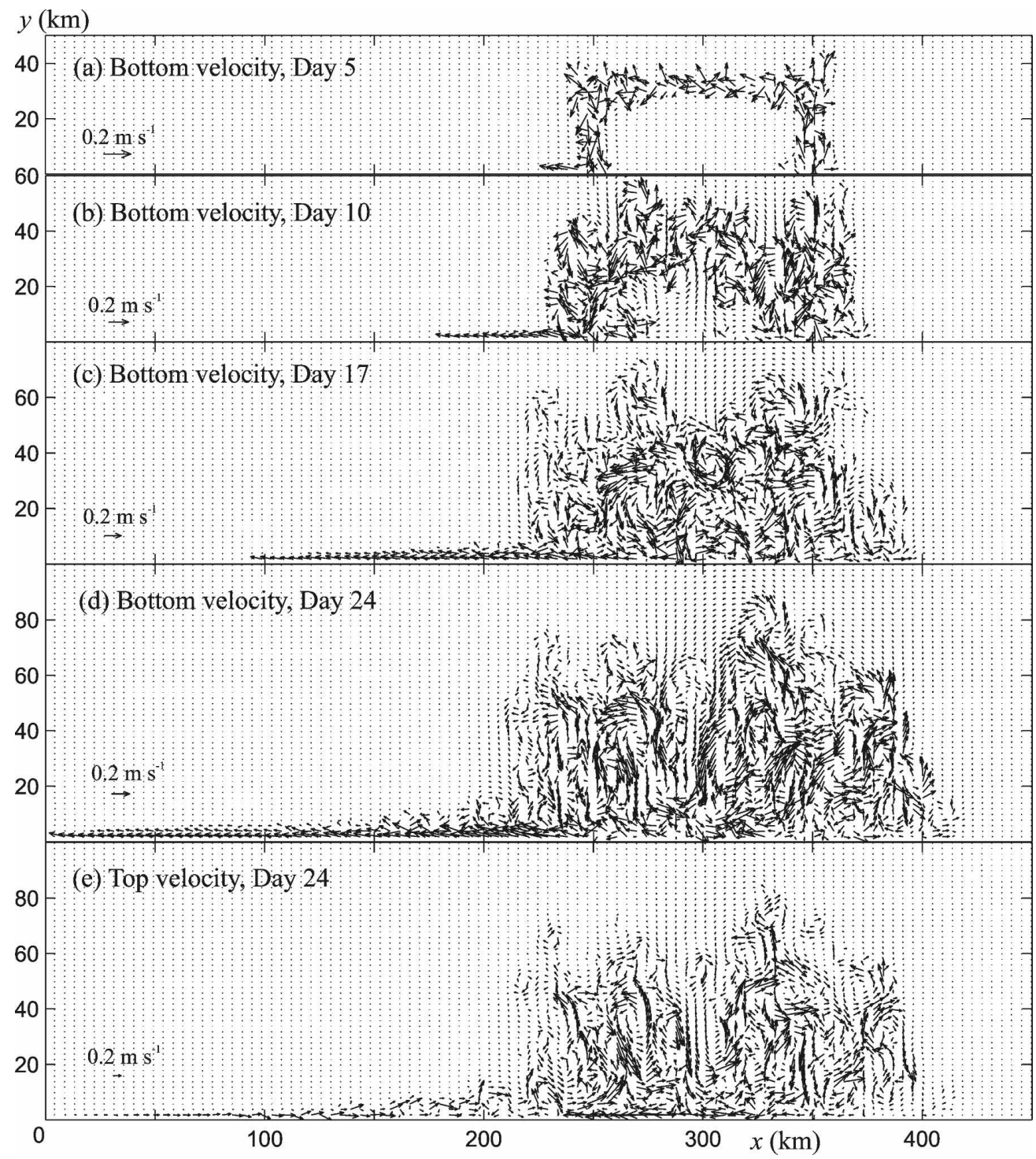

FIG. 4. The bottom and top velocities for the basic case.

of the domain that is $100 \mathrm{~km}$ long to the left of the western polynya edge. We present both the bottom velocities and the salinities at a vertical cross section at $x=180 \mathrm{~km}$. As the salinity anomaly is small at day 10 , we present its distribution at day 11 instead. At day 10 the coastal flow is stable and the cross section of the current is triangular. The current thickens and widens, and at day 13 two perturbations occur around $x=257$ and $x=272 \mathrm{~km}$. By day 17 the first perturbation has moved downstream and split into two "twin" eddies, while the second perturbation is overtaken by a larger eddy in the plume. By this point, the coastal current has thickened sufficiently such that it almost reaches the sea surface. By day 20 the twins have merged into one even bigger eddy, while at the same time one more perturbation has developed around $x=177 \mathrm{~km}$. This latter perturbation removes water from the current and decreases the current thickness. By day 24 the shown part of the current has become fully unstable, and the eddies have made the current cross section nonmonotonic.

The presence of instabilities in a moving boundary current some distance from its front has been observed in rotating tank experiments (Condie and Ivey 1988; Davies et al. 1991). Lane-Serff and Baines (1998) suggested that instability of a current flowing down a sloping bottom could be mainly barotropic in origin and that bottom shear dissipation and vortex stretching in the upper layer play stabilizing and destabilizing roles, respectively, in the eddy-generation process. Ekman 


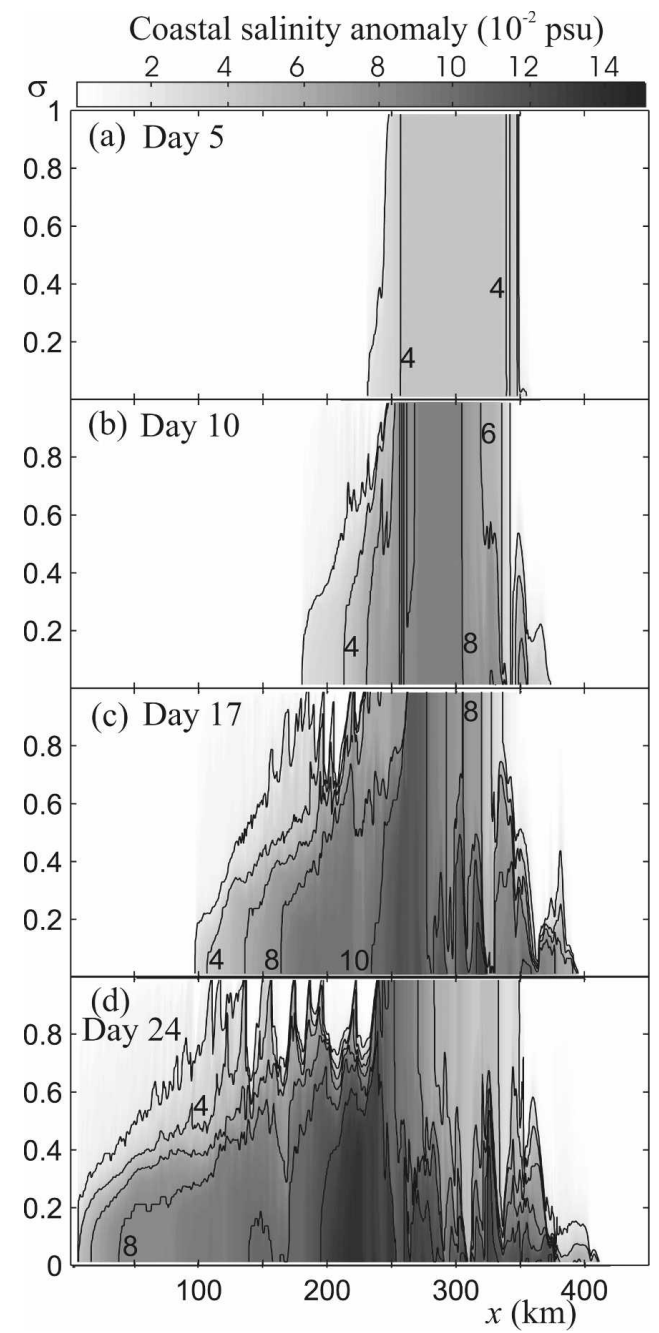

FIG. 5. The salinity anomaly distribution along the coast for the basic case. Contour intervals as in Fig. 2.

drainage was also found to dissipate instabilities in boundary currents (Griffiths and Linden 1981). The vortex tube stretching occurs as the ambient fluid follows the current to greater depth. In our basic case, with constant water depth, the strength of the vortex tube stretching above the current can be described by the ratio of the current thickness to the ambient water depth provided the ambient fluid flows across the current width. Swaters (1991) studied a current flowing along isobaths with bottom slope but without friction and discovered that the destabilizing effect of vortex tube stretching could be overcome by the stabilizing topographic $\beta$-plane effect, which was subsequently supported by the experimental observations of Etling et al. (2000). Sadoux et al. (2000) considered experimental results from a geostrophic current flowing between fluid of different densities and argued that instability of this current is always mixed, that is, both barotropic and baroclinic, with the wall shear driving the barotropic instability. In our experiments, the instability develops only in the upstream part of the current, where the current depth is larger. As the depth of the current increases, there is stronger vortex tube stretching across it until dissipation due to Ekman drainage is overcome and instabilities develop and lead to eddy formation.

From Fig. 6e we see that the perturbation wavelength is around $8 \mathrm{~km}$. The local internal Rossby deformation radius $R$ was calculated by finding the mean density difference and mean current depth over a $50-\mathrm{km}$ area to be approximately $1.4 \mathrm{~km}$, so the nondimensional perturbation wavelength (1) is $\gamma=0.91$. Although this value is somewhat smaller than the experimentally derived mean value of 1.19 (Condie and Ivey 1988), it is still in the range of measured values for moderate Froude numbers with the lower limit being 0.86 . Some other experimental investigations of buoyant currents produced $1.1 \pm 0.3$ (Griffiths and Linden 1982) and $1.18 \pm 0.19$ (Griffiths et al. 1982), where the Rossby radius was determined using the maximum current depth.

\section{Sensitivity studies}

To study sensitivity of the coastal current to different parameters we ran the model with several changes. Only one parameter was changed at a time. Each model run was terminated when the effect of the ocean boundaries became noticeable. The bottom and coastal salinities in the final days of the simulations are presented in Fig. 7. A more detailed picture of the flow pattern in all the currents is shown in Fig. 8, together with the salinity contours at their cross sections about $80 \mathrm{~km}$ upstream from the fronts. Inspecting the plan view given in Fig. 8, we can see that the same two processes of water transport by a plume and a coastal current occur in all simulations.

\section{a. Bottom slope}

In this study we inclined the ocean bottom so that it slopes down offshore with a slope of 0.005 , which Tanaka and Akimoto (2001) identified as the slope leading to the most effective offshore transport. In this case the water depth changes linearly from $400 \mathrm{~m}$ at the coast to $1050 \mathrm{~m}$ at a distance $130 \mathrm{~km}$ off the coast. The run lasted 21 days. The width of the current presented in Fig. 7a looks somewhat bigger than that in the basic case, although this is affected by the presence of a large eddy around $x=170 \mathrm{~km}$. If we compare the current cross section for this case with the basic case (Figs. 8b and $8 d$ ) $80 \mathrm{~km}$ upstream, we see that the width of the current on a sloping bottom is larger than that on a 



FIG. 6. The velocities and cross-section salinity anomaly at $x=180 \mathrm{~km}$ for the basic case coastal current.

horizontal bottom due to the gravity force acting downslope. The coastal salinity contours in this case have zero horizontal gradient upstream (they are almost horizontal). As will be discussed below (see section 5a), the current moves faster with a sloping bottom than in the basic case. While the salinity is higher in the frontal part of the current than in the basic case, due to the faster current upstream the upstream part of the current is not able to accumulate more denser water, so the horizontal salinity gradient is lower. 


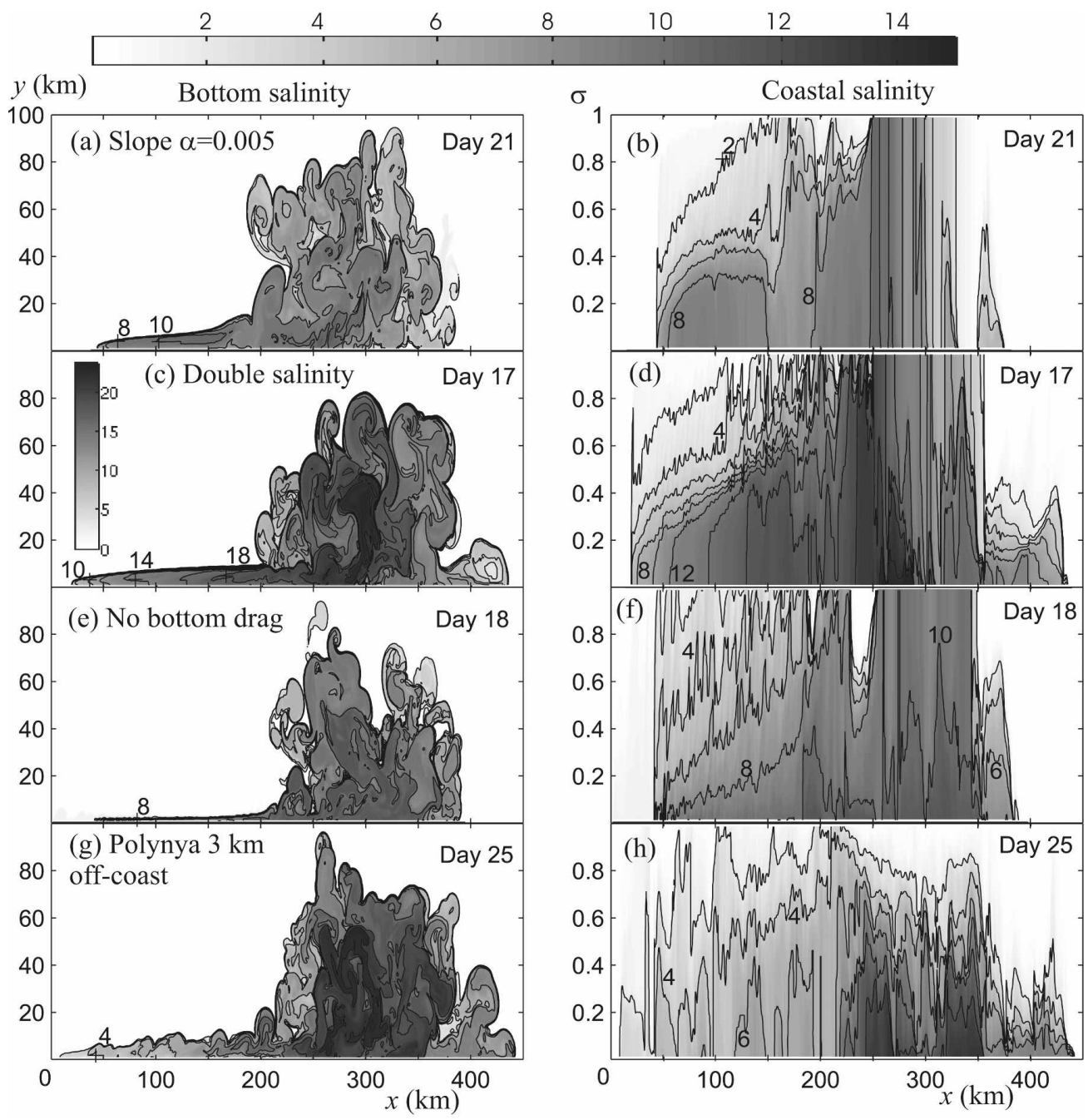

FIG. 7. The bottom and coastal salinity anomaly distribution for the cases of a downward slope of 0.005 , double salinity flux $\left(Q=-8 \times 10^{-5} \mathrm{psu} \mathrm{m} \mathrm{s}^{-1}\right)$, no bottom friction, and polynya position $3 \mathrm{~km}$ offshore: contour intervals as in Fig. 2. (c),(d) The double salinity flux plots have a different grayscale as the salinity is higher than in the other cases.

\section{b. Enhanced salinity flux}

In this study, we doubled the salinity flux into the polynya surface, so it became $Q=-8 \times 10^{-5} \mathrm{psu}$ $\mathrm{m} \mathrm{s}^{-1}$. Due to the higher salinity in this case, it took only 17 days for the current to cross the domain, in comparison with 24 days in the basic case. Inspection of the current cross section in Fig. 8f shows that the width of the current is even bigger than that of the current on a sloping bottom due to a larger pressure drop across it. Within the chosen region shown in Fig. 8e, only minor instabilities can be seen in contrast to the other cases, which may be due to the stabilizing effect of a larger vertical salinity gradient [e.g., Swaters (1991) did not consider stratification within the current itself] and stronger shear dissipation.

\section{c. Zero bottom drag}

In this study, drag at the ocean bottom was removed. In Figs. 7e and 7f, we show the plume and coastal current when drag at the ocean bottom is removed. The coastal current dynamics is somewhat complicated (not shown in the figures): a narrow current propagates quickly until it reaches $x=100 \mathrm{~km}$ at day 12 , then mixes with the ambient fluid leaving only a trace of salinity whose existence can still be seen in terms of background velocities in Fig. 8g; at day 14 a new, stronger current appears, which by day 18 reaches $x=50 \mathrm{~km}$. The width of the current with no bottom drag is between 2 and 3 times smaller than with drag. When there is no bottom drag, the stress near the bottom of the current is more uniform, determining linear velocities 


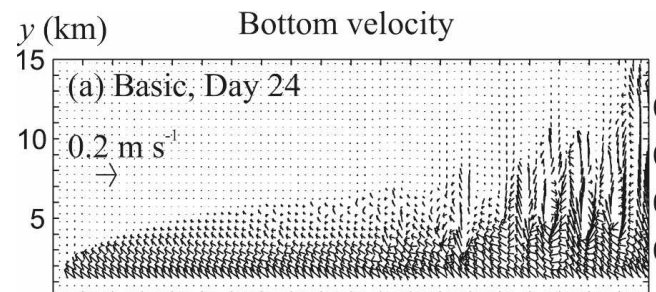

$\sigma_{1}$ Salinity anomaly $\left(10^{-2} \mathrm{psu}\right) 80 \mathrm{~km}$ from front

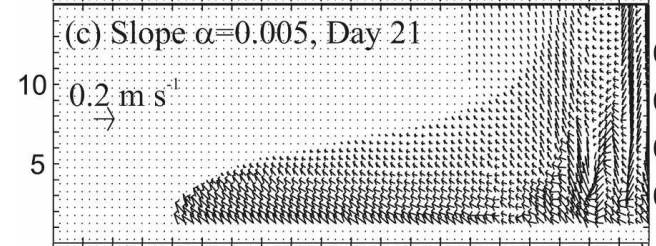

(e) Double salinity flux, Day 17
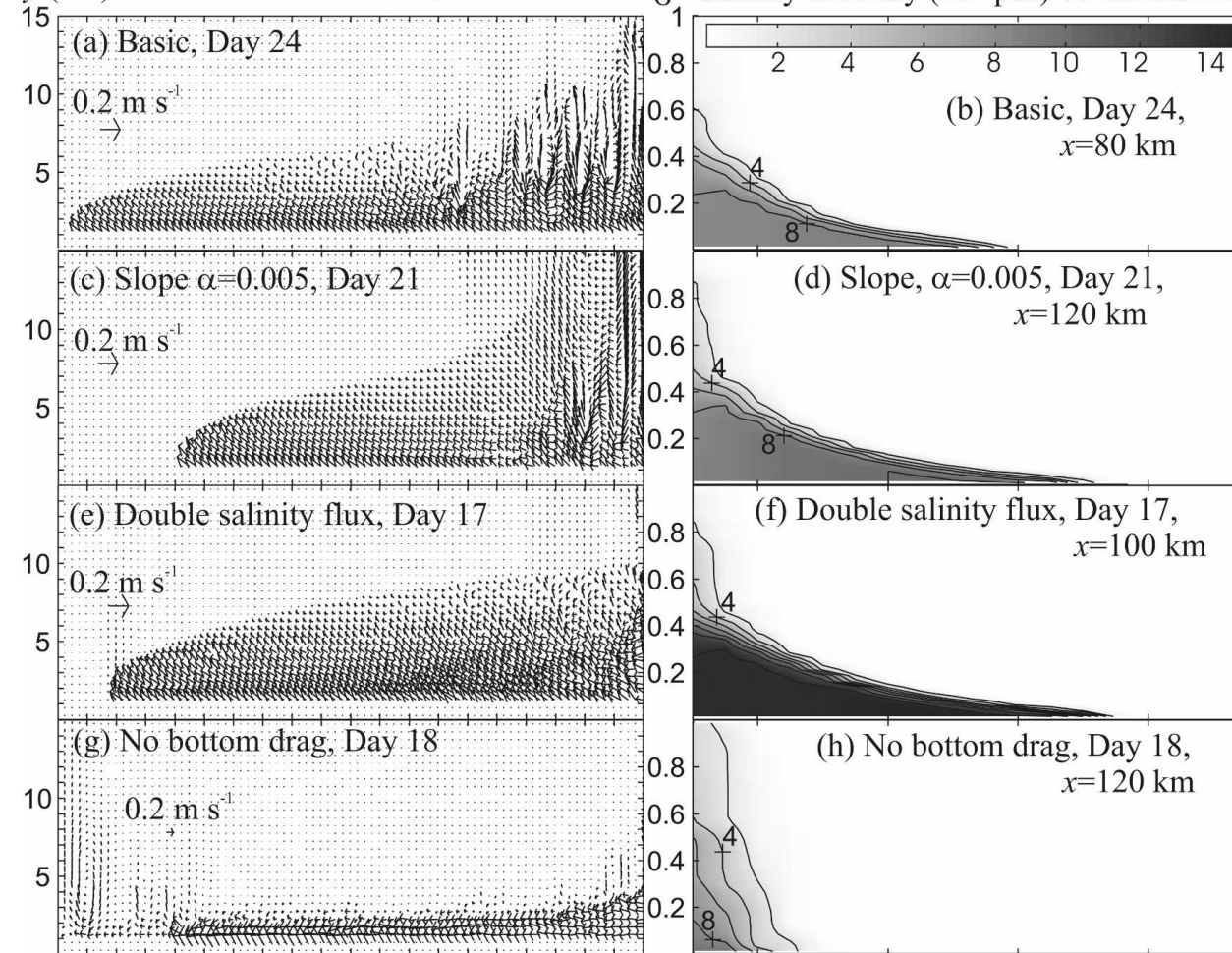

(b) Basic, Day 24,

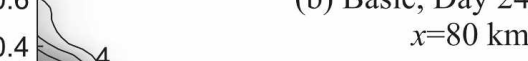

0.2
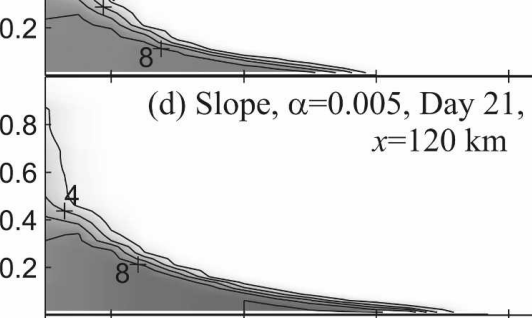

(f) Double salinity flux, Day 17 $x=100 \mathrm{~km}$

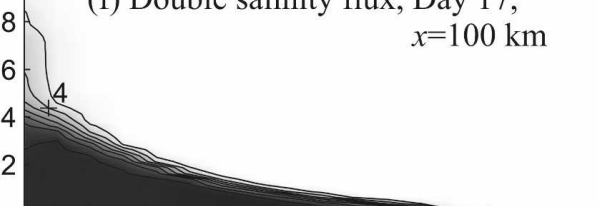

(h) No bottom drag, Day 18 , $x=120 \mathrm{~km}$
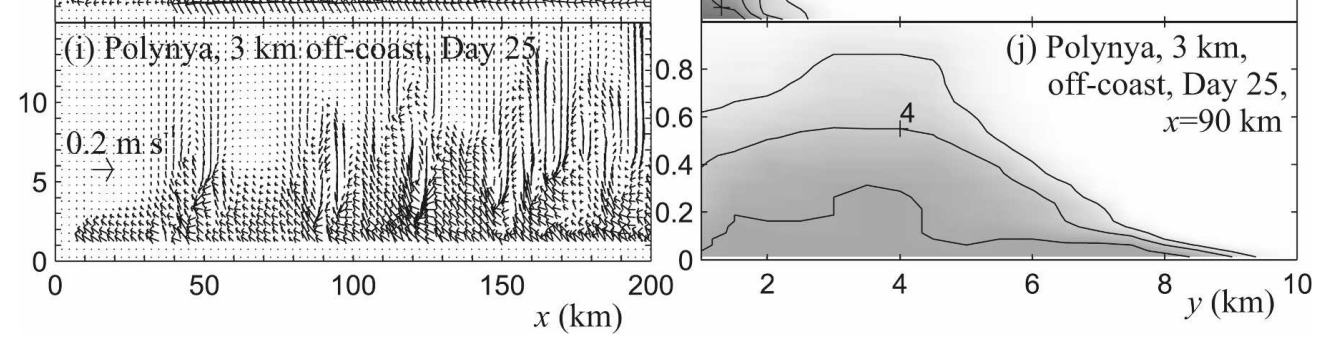

FIG. 8. Comparison between the velocities and salinity anomalies taken at cross sections around $80 \mathrm{~km}$ upstream from the current front for different scenarios.

and salinity contours, and kinetic energy is dissipated only by turbulent mixing [represented by the last term in (2) discussed below].

\section{d. Polynya $3 \mathrm{~km}$ offshore}

In this study, the polynya was shifted $3 \mathrm{~km}$ offshore (Figs. $7 \mathrm{~g}$ and $7 \mathrm{~h}$ ). The run lasted 25 days. The coastal salinity distribution presented in Fig. $7 \mathrm{~h}$ shows that the coastal current is well mixed horizontally and its salinity is less than that of the plume. As can be seen from Fig. $8 \mathrm{i}$, this current is rather unstable, leading to a nonmonotonic salinity distribution across it (Fig. 8j).

The coastal current is permeated with eddies from its outset. Compare the mechanism of the coastal current initiation for the basic case and this case, illustrated in Fig. 9. When the polynya is adjacent to the coast, the motion closest to the coast is anticyclonic (Fig. 9a). When the anticyclonic motion runs into the coast, the velocity across the coast vanishes together with the Coriolis force along the coast, yielding a westward coastal current, driven by the along-coast density gradient. The cyclonic eddy inside the polynya front (Fig. 9e) becomes trapped inside the polynya. By contrast, when the polynya is shifted offshore, the flow generated at the polynya corner (Fig. 9b) is directed southwestward and produces cyclonic motion to the north and anticyclonic motion to the south. The anticyclonic part of the motion again produces a coastal current; however, the southern part of the cyclonic eddy to the north is carried westward by the current. The succession of anticyclonic and cyclonic eddies reaching the coast causes the coastal current to consist of both eddies and stable flow.

\section{e. Polynya $15 \mathrm{~km}$ offshore}

In our previous examples, the coastal current was generated close to the polynya. As the model uses a 

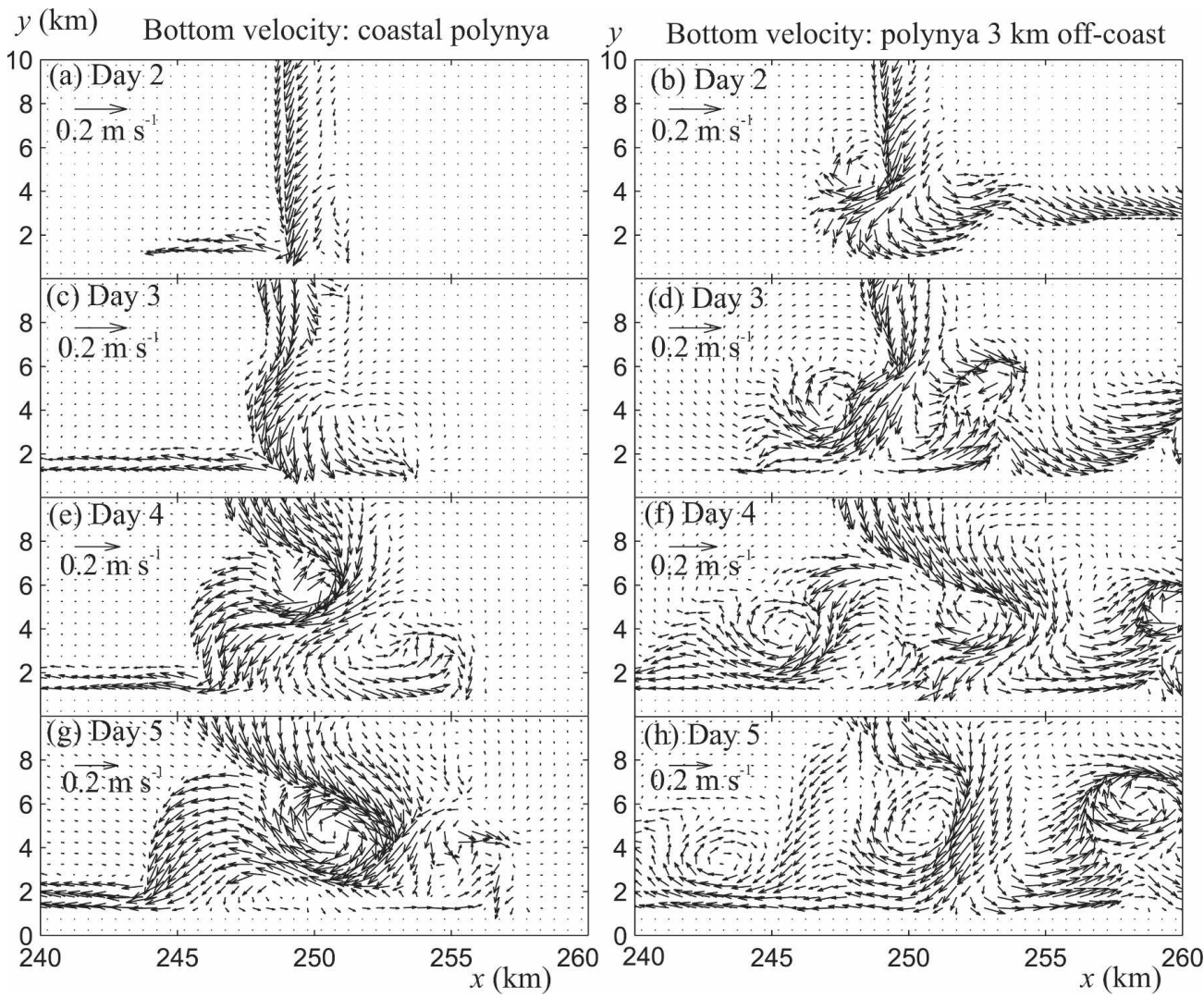

FIG. 9. The initiation of the coastal current for (left) the basic case and (right) the case with the polynya situated $3 \mathrm{~km}$ offshore.

convective adjustment scheme, it is necessary to ascertain that it is not this scheme that is responsible for the coastal current formation, as the calculations of Gawarkiewicz and Chapman (1995) did not produce a coastal current. To test this, we ran the model with the polynya positioned $15 \mathrm{~km}$ offshore with both a flat bottom and an offshore downward sloping bottom with a slope of 0.005 ; the bottom salinity for these cases is plotted in Fig. 10. When the bottom is sloping, we took the depth of $400 \mathrm{~m}$ to be not at the coast as before, but $15 \mathrm{~km}$ offshore where the polynya begins, to make the comparison with the basic case more meaningful. We plot results at days 11 and 12 to capture the moment when the coastal current is formed for the flat and sloping bottom cases, respectively. Although with a sloping bottom the plume has to move upslope to reach the coast, both the flat and sloping bottom scenarios are quite similar: once the plume reaches the coast, a coastal current is formed. The upslope motion is possible because the ocean depth decreases only by $75 \mathrm{~m}$ as the dense water covers $15 \mathrm{~km}$ and reaches the coast, while the plume thickness is on the order of the ocean depth itself (this can be seen from the basic case along- coast salinities in Fig. 5b) so that the horizontal buoyancy gradient force that drives the flow south of the polynya persists. The boundary current is not formed on the southern part of this polynya as its formation requires a sharp increase in the bottom slope. Because the coastal current is formed not within or immediately adjacent to the polynya in these cases, we can be sure that convective adjustment is not responsible for the coastal current formation. As the coastal current density is smaller than the basic case because the polynya is removed from the coast, the coastal current speed is less. The coastal current is also quite unstable because it forms in an unstable environment, as was shown for the 3-km offshore polynya.

When the polynya is situated offshore, the dense water reaches the coast in the form of eddies. Several studies (e.g., Nof 1988a,b; Shi and Nof 1994; Sansón et al. 1998), interpreted for our Southern Hemisphere study, show that, if a single eddy encounters the coast, then an anticyclonic eddy would produce a westward boundary current. The eddy itself can move along the coast owing to a delicate balance of the topographic $\beta$-plane effect-the so-called rocket effect describing 
Bottom salinity anomaly $\left(10^{-2} \mathrm{psu}\right)$. Polynya $15 \mathrm{~km}$ off-coast

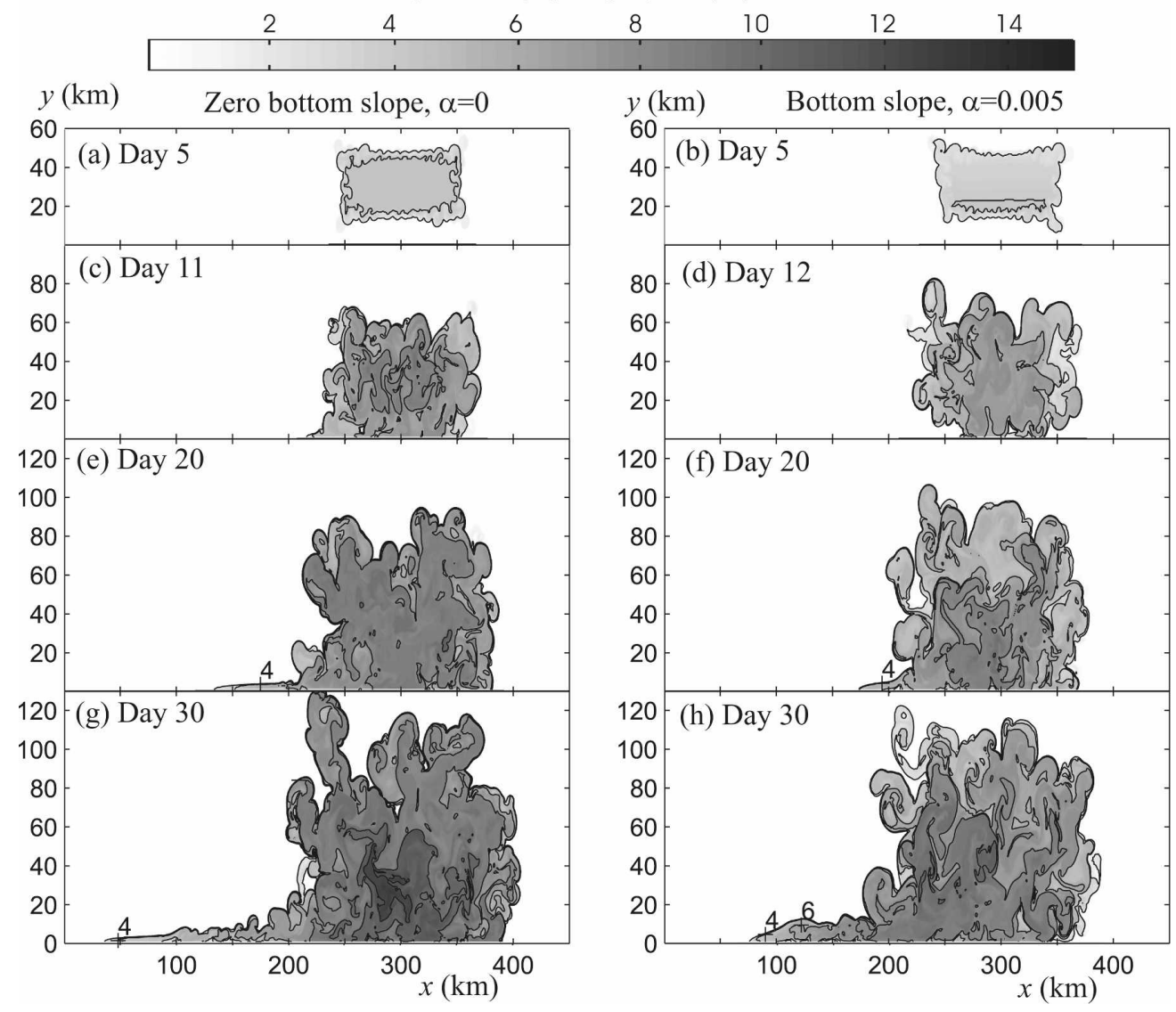

FIG. 10. Evolution of the bottom salinity anomalies for polynyas situated $15 \mathrm{~km}$ offshore on (left) zero bottom slope and (right) a downward slope of 0.005 .

the linear momentum change of the eddy as it sends a jet along the coast-and the image effect due to symmetry about the wall. The image effect was found by Shi and Nof (1994) to dominate the eddy motion in the direction opposite to the boundary current flow. A more detailed study by Sansón et al. (1998) with different strengths of the topographic $\beta$-plane effect and eddy vorticity showed that in some cases the eddy moves initially in one direction along the boundary and then to the other. In our calculations the situation is more complicated as several eddies interact with each other. A close-up on the bottom velocity near the wall shows that the eddies reaching the wall are anticyclones, so generation of a westward coastal current concurs with the previous study results.

\section{f. Lower-resolution calculation}

To try to understand why Gawarkiewicz and Chapman (1995) did not observe any coastal current, we ran the model with a lower-resolution grid, $2 \mathrm{~km}$ in the horizontal with $20-\mathrm{m}$ vertical resolution (20 sigma lay- ers), and an 18-s barotropic and 900-s baroclinic time step (Fig. 11). After 24 days, which was enough for the basic case current to cover $200 \mathrm{~km}$, the low-resolution current covered only $50 \mathrm{~km}$ and its salinity anomaly was almost two times smaller than that of the plume. Such a current can easily be taken for an eddy. The distribution of the coastal salinity shows only weak asymmetry of its distribution. It should be noted that our barotropic time step was more than an order of magnitude smaller than that adopted by Gawarkiewicz and Chapman (1995) due to the explicit nature of the numerical algorithm in POLCOMS. Although the spatial resolution used by Gawarkiewicz and Chapman $(1.03 \mathrm{~km})$ was only 2 times coarser than our high-resolution run, because the models have different numerical schemes a direct comparison of grid resolutions is not meaningful; here we show only that a relative decrease in the model resolution leads to a poorer coastal current simulation. It could be argued that another possible reason why Gawarkiewicz and Chapman did not observe the current lies in their use of periodic boundary conditions 

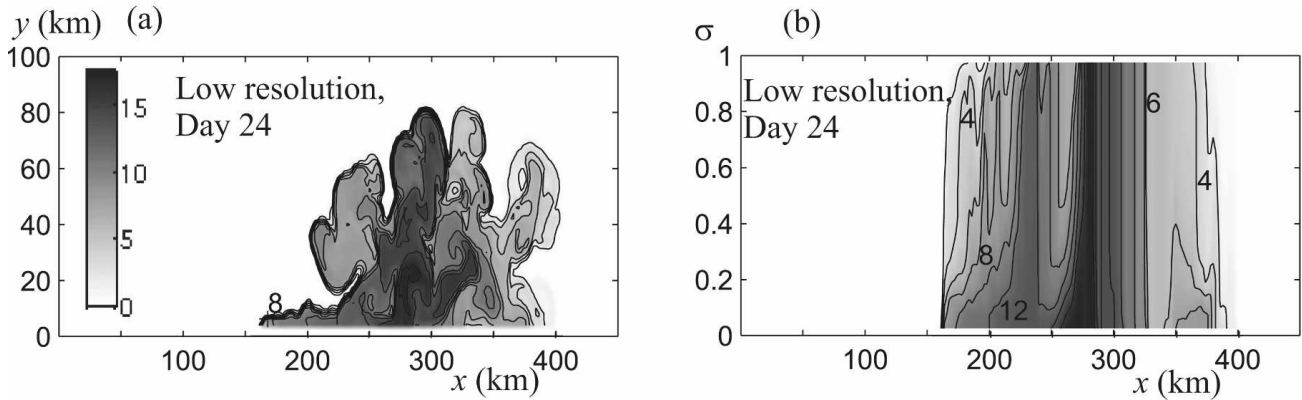

FIG. 11. The basic case bottom and coastal salinity anomaly $\left(10^{-2} \mathrm{psu}\right)$ for a lower-resolution grid of 2 $\mathrm{km}$ horizontally and $20 \mathrm{~m}$ vertically.

at the eastern and western domain boundaries that sustain a zero net pressure gradient along the coast. However, because in our basic case calculation the net pressure gradient is zero up to day 8 , by which time the current has fully formed, the difference in the boundary conditions cannot be the reason behind this discrepancy. Another difference between the model used by Gawarkiewicz and Chapman (SPEM) and ourselves is that SPEM used a rigid lid approximation, while POLCOMS treats the ocean surface as a free surface. However, as the main driving force for the plume and current is the buoyancy difference between the forcing region and the ambient water, it is unlikely that the rigid lid approximation could be responsible for the absence of the coastal current modeled by SPEM. Based on these arguments, we believe it is reasonable to conclude that a high-resolution grid is necessary to model the formation of the coastal current.

\section{Discussion}

\section{a. Comparison of coastal current front characteristics}

We calculated the mean speed and width of the coastal current over a $50-\mathrm{km}$ length starting $1 \mathrm{~km}$ upstream from the front of the current. Because the case of zero bottom drag was poorly resolved in our calculations due to the small width of the current, its data were highly scattered and are not given here. In Figs. $12 \mathrm{a}$ and $12 \mathrm{~b}$, we show the mean speed $V$ normalized by the gravity-inertia wave speed $V_{b}=\left(g^{\prime} h\right)^{1 / 2}$ as well as the mean width $D$ normalized by the Rossby radius $R=$ $\left(g^{\prime} h\right)^{1 / 2} / f$. We plot the data beginning from the first day when the current length is longer than $50 \mathrm{~km}$. This occurred on day 10 for the basic case, day 12 for the case with bottom slope, day 7 for the case with double surface salinity flux, and the day 13 for the case when the polynya is $3 \mathrm{~km}$ offshore. The results for the offshore polynya are much more variable than the other cases owing to its unstable flow and, for this reason, results in which the polynya was placed $15 \mathrm{~km}$ offshore are not considered. For the coastal polynya cases, the normalized speed ranges between 0.3 and 0.5 , on average decreasing with time. The time-averaged values of $V / V_{b}$ are 0.412 for the basic case, 0.42 for the sloping bottom, 0.388 for the double salinity flux, and 0.47 for the offshore polynya. The mean normalized width tends to increase with time, in accordance with the experimental results of Condie and Ivey (1988). The normalized current width is estimated as $1.9 \pm 0.3$. This is somewhat smaller than $2.4 \pm 0.5$ given by an isotherm that is one-eighth of the maximum temperature anomaly in the experiments of Condie and Ivey, although the experiments are not an exact analog of the modeled current. The time-averaged values for the normalized current width were 1.84 for the basic case, 1.87 for the sloping bottom, 1.79 for the double salinity flux, and 2.08 for the offshore polynya.

The plots of nonnormalized buoyancy (reduced gravity), speed, width, depth, gravity-inertia wave speed, and Rossby radius averaged over the frontal $50 \mathrm{~km}$ of the currents are presented in Figs. 12c-h, respectively. If we compare the horizontal salinity gradients for the basic case (Fig. 5) at different times, then we can conclude that the gradient increases in time; that is, the denser water situated upstream moves faster than the less-dense water in the frontal part. This causes the buoyancy of the frontal $50 \mathrm{~km}$ to increase, on average, with time in all cases.

The presence of a downward slope produces an additional offshore force owing to a higher upper-surface slope and hence a higher horizontal pressure gradient that drives the denser water slightly further offshore making the current wider. During the initial period of the dense water spreading away from the coast, the coastal current is slower than in the basic case. In particular, although the front of the current on the slope leaves the polynya edge (at $x=250 \mathrm{~km}$ ) one day later than in the basic case (which leaves the polynya on day 3 ), due to the extra depth of the shelf with the sloping 
(a) Normalized current speed

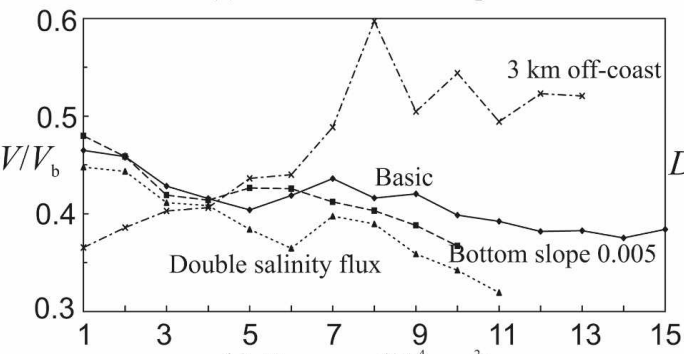

(c) Buoyancy $\left(10^{-4} \mathrm{~m} \mathrm{~s}^{-2}\right)$

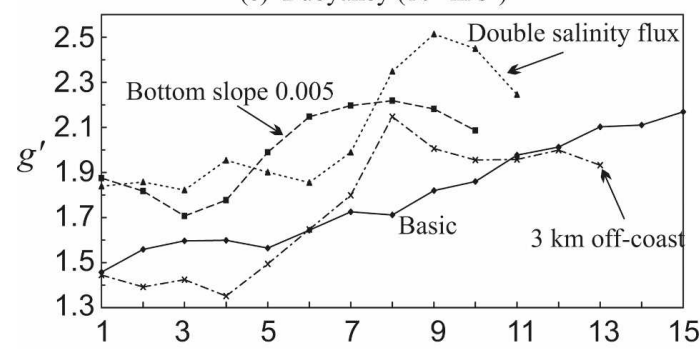

(e) Current width $(\mathrm{km})$

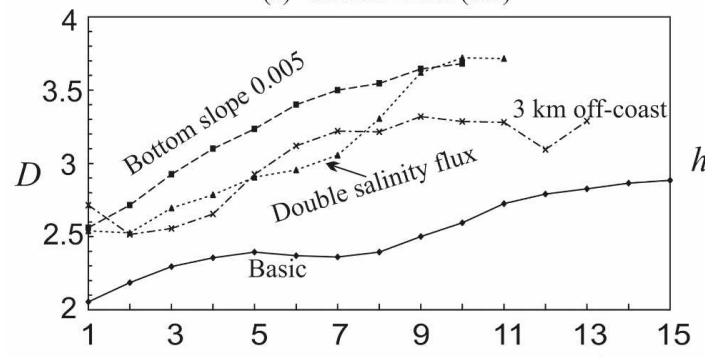

(g) Gravity-inertia wave speed $\left(\mathrm{cm} \mathrm{s}^{-1}\right)$

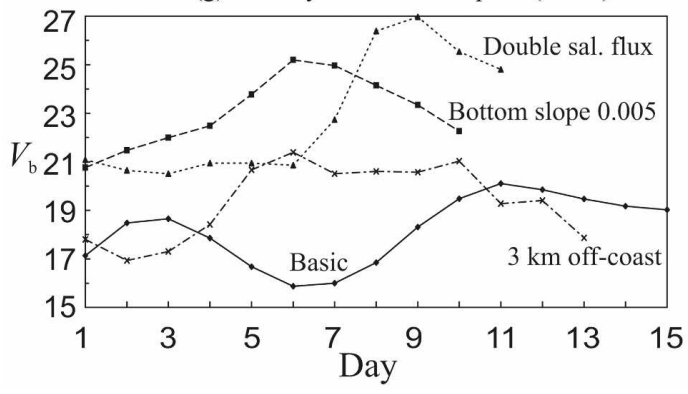

(b) Normalized current width

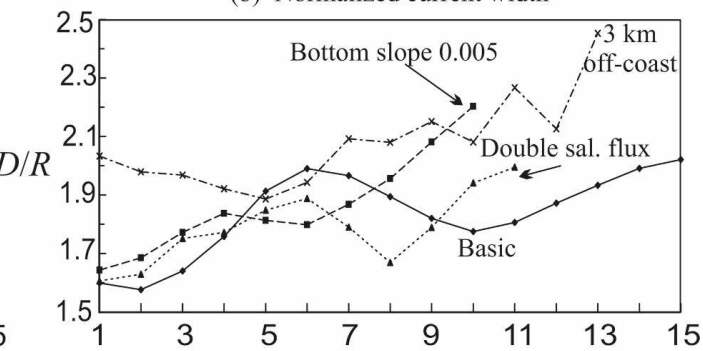

(d) Current speed $\left(\mathrm{cm} \mathrm{s}^{-1}\right)$

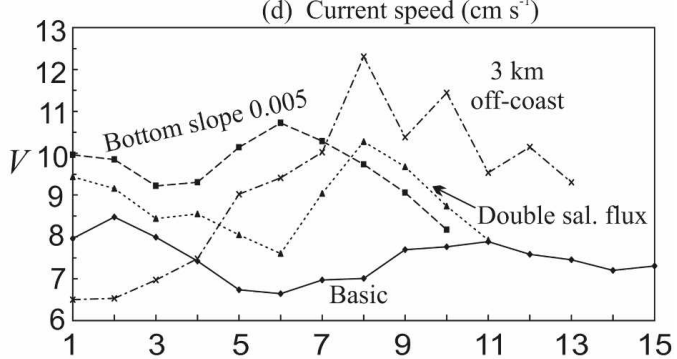

(f) Current depth (m)

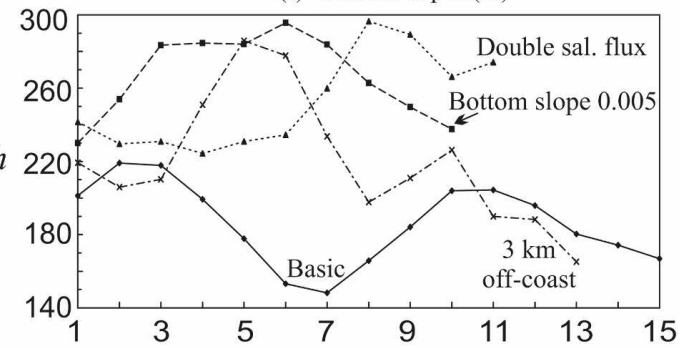

(h) Rossby radius $(\mathrm{km})$

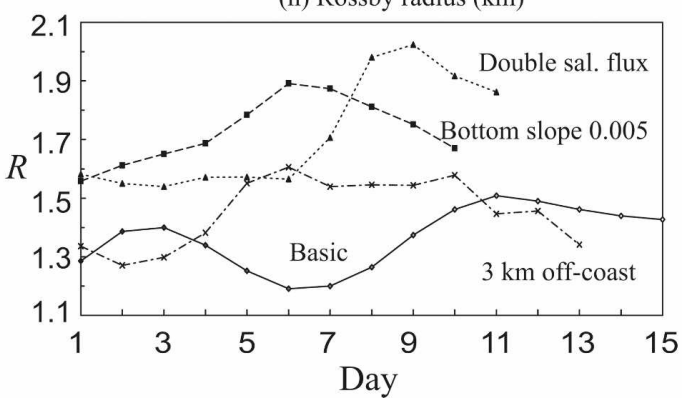

FIG. 12. The normalized speed, width, and nonnormalized other characteristics of the current averaged over a $50-\mathrm{km}$ frontal zone.

bottom, during the next five days the basic current covers $49.4 \mathrm{~km}$, while the current on the slope covers only $33.2 \mathrm{~km}$. By day 10, the front of the current on the sloping bottom reaches only $x=206 \mathrm{~km}$, lagging $26 \mathrm{~km}$ behind the basic case current whose front is at $x=180.4$ $\mathrm{km}$ (Fig. 2). This initial period of slow motion allows salinity to accumulate, which then drives the current more rapidly along the coast (moving on average 1.3 times faster than in the basic case), so that the additional offshore gravitational force is balanced by the Coriolis force.

When the salinity flux is doubled, the coastal current is still formed at low salinity values and its exterior part is determined by the 0.02 psu salinity anomaly (Figs. $7 \mathrm{~d}$ and $8 \mathrm{f}$ ). However, as higher salinity water is supplied, this leads to a greater along-coast salinity gradient so that the mean density in the $50-\mathrm{km}$ frontal part of the current is larger than in the basic case. This leads to a higher speed and wider current as the pressure difference driving the current both along the coast and offshore increases.

The buoyancy evolution of the coastal current formed from the offshore polynya is similar to the basic case. The similarity of the buoyancy evolution is caused 
by the similar salinity flux and the bottom geometry, even though the salinity distribution is quite different (Figs. 5d and 7h). With regard to all other parameters, the offshore polynya produces rather variable values owing to the unstable flow.

The depth of the current in the case of a downward bottom slope and double salinity flux is larger than the basic current depth (the limits of the current are determined by the 0.02 -psu salinity anomaly). From Fig. 5 it is seen that the denser water near the bottom penetrates under the lighter water so that, when the mean density is higher, a deeper current is produced. The presence of vertical mixing also deepens the current, and the depth of the coastal current produced by the offshore polynya is high due to the strong mixing caused by its unstable nature.

The gravity-inertia wave speed and the Rossby radius plots are similar, as these parameters are proportional to each other. As the density and depth of the coastal current on the sloping bottom and with a double salinity flux are on average larger than in the basic case, the gravity-inertia wave speed and Rossby radius are also larger. The relative evolution of these parameters in the basic current and with an offshore polynya current is similar.

\section{b. Bottom drag effect}

Our calculations have shown that the variation of bottom drag has a significant impact on the current width; therefore, we study here the bottom drag effect in more detail. If we assume a geostrophic balance across the coastal current, then $|f| u=g^{\prime} \partial h / \partial y$ so that, if the along-coast speed $|u|$ increases owing to a reduction of bottom friction, then with no appreciable change in the reduced gravity, the thickness gradient increases by the same factor, leading to a narrower current.

In Fig. 13 we present the cross-sectional distribution of the along-coast velocity $u$, across-coast velocity $v$, and the salinity anomaly for the quadratic law drag coefficient $C$ taken as $0,0.001,0.003$, and 0.005 . The cross sections are taken about $80 \mathrm{~km}$ from the fronts from the last day of the calculations. For $C<0.005$, the dynamics of the coastal current is similar to that when there is no bottom drag: a narrow current propagates quickly until it mixes with the ambient fluid, leaving only a trace of salinity. Then a new, stronger current appears that eventually overtakes the first current.

From Fig. 13a it can be seen that the current without bottom drag consists of an internal core, where the along-coast velocity is almost homogeneous, and a peripheral region above and to the right (offshore) of the core where the velocity gradient is higher due to mixing with the ambient fluid and the no-slip condition at the coast. The current profile is seen to consist of two parts: a higher slope region above the core and and a lower slope region to the right of the core. If geostrophic equilibrium is assumed, higher velocities require a greater slope of the current profile. As the drag is increased to $C=0.001$, the along-slope velocity decreases at the bottom, tending to retard the core flow, and the velocity gradient in the peripheral part decreases. Geostrophy implies that a slower flow requires a smaller current depth gradient, so the current widens. Also, the presence of bottom drag now leads to an off-coast Ekman flow, which is seen up to about $40 \mathrm{~m}$ from the bottom, which widens the current. If the bottom drag is increased further, up to $C=0.005$, the along- $y$ velocity gradient near the bottom becomes more uniform, which leads to a smaller difference between the uppersurface slope of the current above the core and the slope of the region to the right of the core, which is also supported by calculations with $C=0.007$ (not shown). The slowing down of the current is evident from Table 1 , where the main characteristics of the current on the last day of the runs are shown. From the table, we see in quantitative terms that, as the drag increases, the current becomes wider, shallower, and slower.

\section{c. Vertical eddy diffusivity and viscosity}

The strength of the bottom drag effect depends on momentum transfer by vertical mixing. The vertical diffusivity of momentum (viscosity) and salinity, $K_{z}$, is determined in POLCOMS using the Mellor-YamadaGalperin level-2.5 turbulent closure (Mellor and Yamada 1974; Galperin et al. 1988) with an algebraic mixing length $l$. The evolution of twice the turbulent kinetic energy per unit mass $q^{2}$ is given by

$$
\frac{\partial q^{2}}{\partial t}=\frac{\partial}{\partial z} K_{q} \frac{\partial q^{2}}{\partial z}+2 K_{z} M^{2}-2\left(K_{h}-\alpha K_{z}\right) N^{2}-\frac{2 q^{3}}{B_{1} l},
$$

where $B_{1}=16.6, \alpha=0.7$

$$
M^{2}=\left(\frac{\partial u}{\partial z}\right)^{2}+\left(\frac{\partial v}{\partial z}\right)^{2}, \quad N^{2}=\frac{\partial b}{\partial z},
$$

and $b\left(=g^{\prime}\right)$ is buoyancy. The term $\alpha K_{z}$ models vertical mixing by long internal waves (Mellor 1989) and the diffusivities are given by

$$
K_{z}=S_{m} l q, \quad K_{h}=S_{H} l q, \quad K_{q}=S_{q} l q,
$$

which use the stability relations from Galperin et al. (1988): 


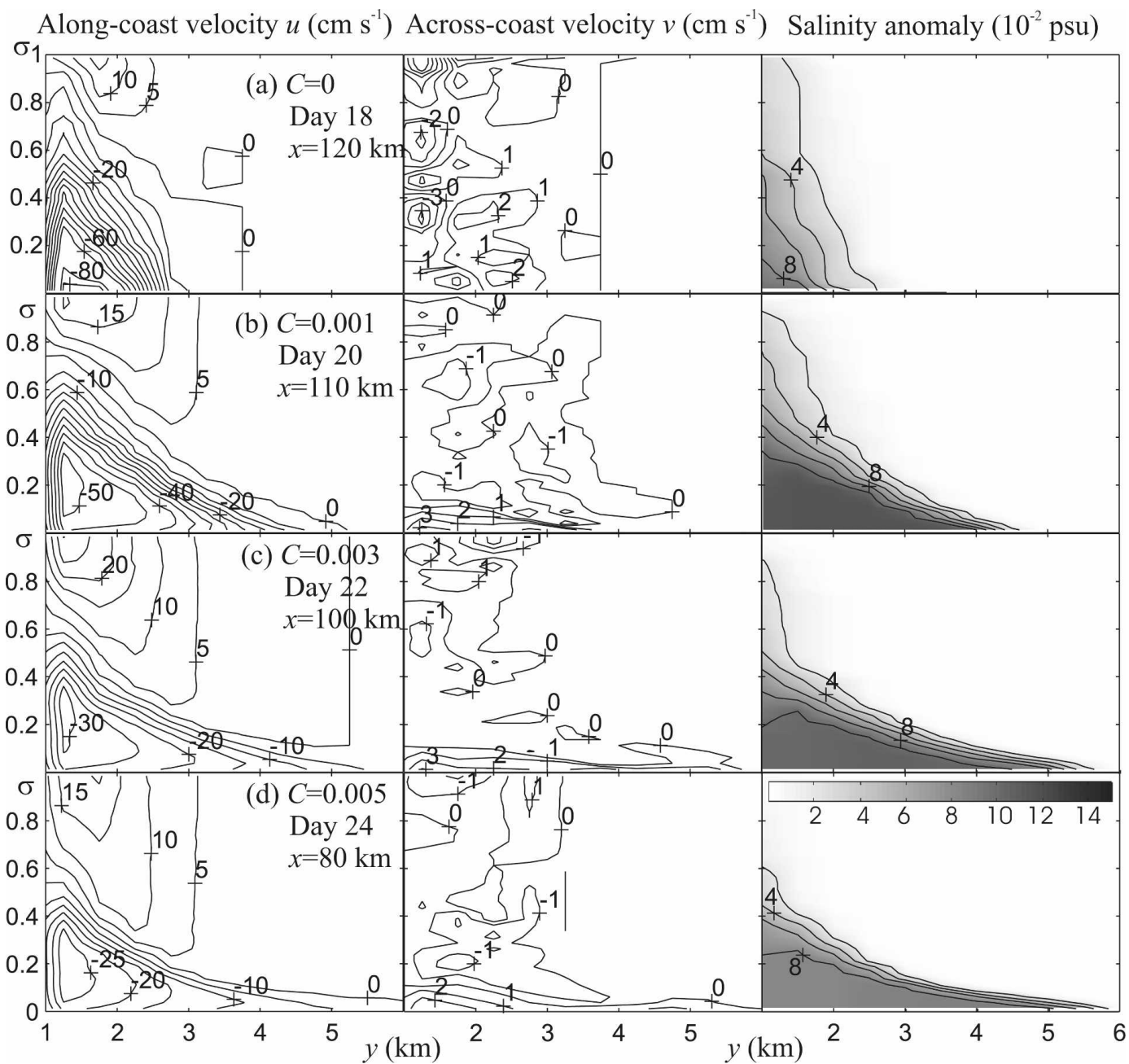

FIG. 13. The (left) along-coast velocity, (middle) across-coast velocity, and (right) salinity anomaly for different bottom drag coefficients $C, 80 \mathrm{~km}$ from the current front.

$$
\begin{aligned}
S_{m} & =\frac{0.3933-3.086 G}{(1-34.68 G)(1-6.127 G)}, \quad S_{h}=\frac{0.4939}{1-34.68 G} \\
S_{q} & =2
\end{aligned}
$$

with $G=-l^{2} N^{2} / q^{2}$.
Owing to the inherent uncertainty in modeling turbulence, here we study how increasing and decreasing $S_{m}, S_{h}$, and $S_{q}$ by a factor of 5 would affect the coastal current. The current frontal characteristics on the last day of the calculations are shown in the bottom part of Table 1. In Fig. 14 we show the cross sections of the

TABLE 1. The 50-km-averaged frontal current characteristics at the last day of the runs with varied bottom drag and vertical viscosity and eddy diffusivity.

\begin{tabular}{ccccccccc}
\hline \hline $\begin{array}{c}C \\
\left(\times 10^{-3}\right)\end{array}$ & $\begin{array}{c}\text { Date } \\
(\text { day })\end{array}$ & $\begin{array}{c}V / V_{b} \\
-\end{array}$ & $\begin{array}{c}D / R \\
-\end{array}$ & $\begin{array}{c}g^{\prime} \\
\left(\times 10^{-4} \mathrm{~m} \mathrm{~s}^{-2}\right)\end{array}$ & $\begin{array}{c}V \\
\left(\mathrm{~cm} \mathrm{~s}^{-1}\right)\end{array}$ & $\begin{array}{c}D \\
(\mathrm{~km})\end{array}$ & $\begin{array}{c}h \\
(\mathrm{~m})\end{array}$ & $\begin{array}{c}V_{b} \\
\left(\mathrm{~cm} \mathrm{~s}^{-1}\right)\end{array}$ \\
\hline 0 & 18 & 1.1 & 0.4 & 2.38 & 32.8 & 0.87 & 369 & 29.6 \\
1 & 20 & 0.49 & 1.0 & 2.13 & 12.6 & 1.94 & 308 & 25.6 \\
$(\mathrm{~km})$ \\
3
\end{tabular}




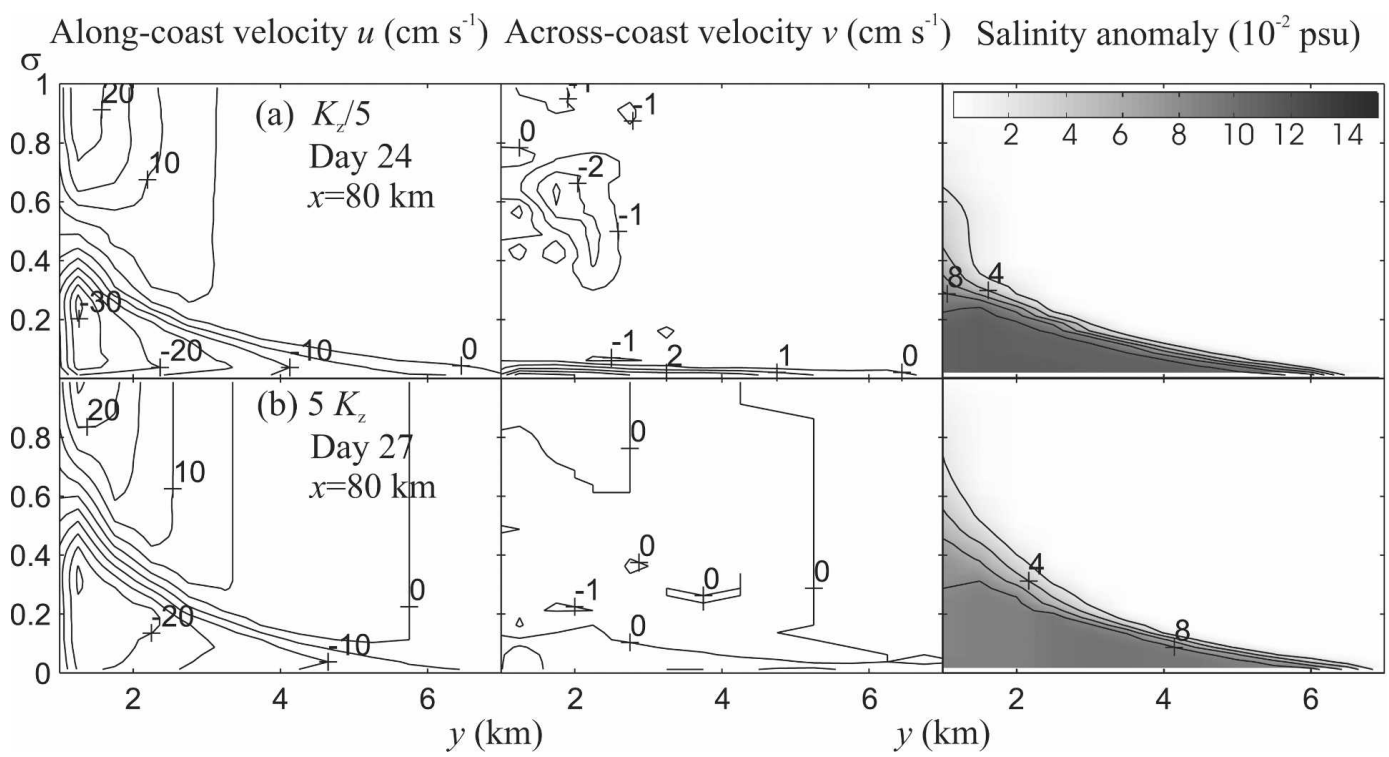

FIG. 14. Same as in Fig. 13, but for 5 times (top) smaller and (bottom) larger eddy diffusivities, at the cross section $80 \mathrm{~km}$ from the current front.

currents in terms of velocities and salinity anomaly around $80 \mathrm{~km}$ from the front. Although the mean speed $V$ of the basic case current is slightly greater than the speed of the current with the eddy diffusivities reduced by a factor of 5 , by day 24 both currents reach $x=6.2$ and $x=6.1 \mathrm{~km}$, respectively (so that the basic current is slower), while the current with the eddy diffusivities enhanced by a factor of 5 reached $x=3.3 \mathrm{~km}$ only by day 27. The mean frontal speed of the reduced eddy diffusivity current appears smaller because it is found from averaging within the current body delineated by the salinity anomaly contour 0.02 psu; from Fig. 14, it can be seen that in the case of decreased eddy diffusivity, part of the current adjacent to the coast occupies the region of backflow, probably due to weaker mixing. Furthermore, while at the cross section the lowest eddy diffusivity current has the highest salinity (Fig. 14), its buoyancy is smaller in the frontal 50-km-long part (Table 1) as the 0.1 psu salinity anomaly layer starts around $50 \mathrm{~km}$ from the front. In cases of the basic current and the current with the largest eddy diffusivity, the 0.1-psu layers start 130 and $110 \mathrm{~km}$ from their fronts, respectively.

As can be seen from the contour plots of velocity 80 $\mathrm{km}$ from the current front with altered diffusivitites (Fig. 14) compared with the basic case (Fig. 13), the most obvious effect of increasing the eddy diffusivities is to increase the depth of the bottom shear layer and, consequently, to decrease the along-coast velocities. The fact that increasing the eddy diffusivities by a factor of 5 alters the basic current speed to a greater de- gree than reducing the eddy diffusivities by a factor of 5 may be interpreted by noting that the bottom shear layer does not extend fully into the fastest inner core of the current in the basic case. A reduction in the eddy diffusivities reduces the size of the shear layer, but this only partly affected the inner core in the basic case as the effect on current speed is only moderate. On the other hand, increasing the eddy diffusivities extends the shear layer fully into the inner core of the current, which thus causes a relatively large reduction in current speed.

\section{d. Salt flux balance}

Numerical and laboratory studies have shown that a buoyancy flux over a limited area can eventually be balanced by buoyancy removal by eddies generated by baroclinic instability (Legg and Marshall 1993; Ivey et al. 1995; Brickman 1995). Visbeck et al. (1996) developed a model to study the balance between a surface buoyancy flux over a circular region and buoyancy removal by eddies whose kinetic energy comes from the potential energy released as they subside from the forcing region. For an initially unstratified ambient fluid where the mixed region reaches the bottom, Visbeck et al. (1996) assumed that mixing by eddies outside the mixed region (which has the size of the forcing region) eventually results in a stratified ambient that ensures equilibrium. In this case, the equilibrium buoyancy $b_{e}$ in the mixed region is given as

$$
b_{e} \approx 16 \frac{\left(B_{0} r\right)^{2 / 3}}{H},
$$


(a)

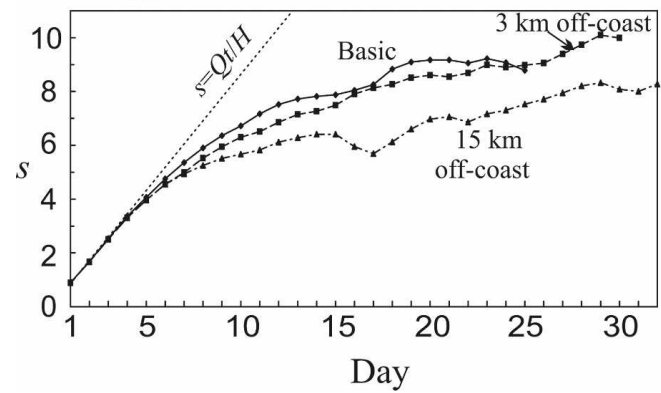

(b)

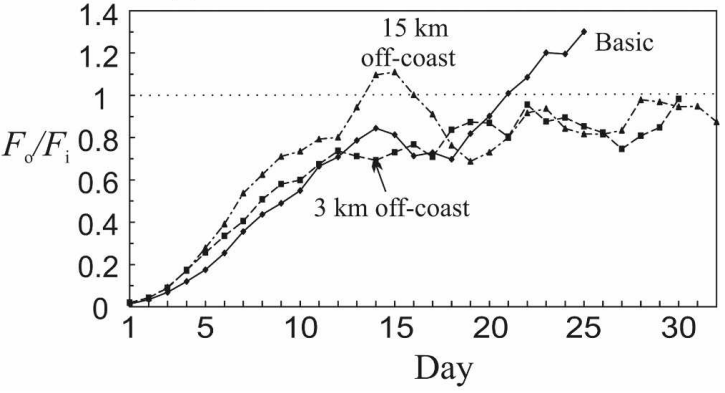

FIG. 15. (a) The mean salinity anomaly under the polynya $\left(10^{-2} \mathrm{psu}\right)$ and (b) the ratio of the salinity outflux through its perimeter $F_{o}$ to the influx from the polynya surface $F_{i}$.

where $B_{0}$ is the surface buoyancy flux, $r$ is the radius of the forcing region, and $H$ is the water depth. For our basic case, we take $B_{0}=3 \times 10^{-7} \mathrm{~m}^{2} \mathrm{~s}^{-3}, r=30 \mathrm{~km}$, and $H=400 \mathrm{~m}$, which determine $b_{e}=1.7 \times 10^{-3} \mathrm{~m} \mathrm{~s}^{-2}$, equivalent to a 0.22 psu salinity anomaly. Visbeck et al. (1996) calculated the time to attain the equilibrium state as

$$
t_{e} \gtrsim 16 \frac{r^{2 / 3}}{B_{0}^{2 / 3}},
$$

which for our basic case is 27 days. In Fig. 13a we show the mean salinity evolution under the polynya, and Fig. $13 \mathrm{~b}$ shows the ratio of the salinity loss at the polynya perimeter $F_{o}$ to the cumulative salinity flux into the polynya $F_{i}$ for the basic case as well as when the polynya is 3 and $15 \mathrm{~km}$ offshore. We plot all available data here because, even though for the later stages of the basic case and $3-\mathrm{km}$ offshore polynya simulations the coastal current reaches the westernmost domain boundary and turns north, this does not affect the nearpolynya dynamics. As the salinity flux ratio is greatly variable, we plot five-day means. The dotted line $s=$ $Q t / H$ in Fig. 15a shows the mean salinity evolution when no salinity loss is assumed. As previously discussed, the horizontal salinity transport becomes important after five days, while before that $s=Q t / H$ holds very well. The coast prevents salinity transport by eddies southward; therefore the mean polynya salinity decreases with the polynya distance from the coast. The maximum salinity for the shown period is about $0.1 \mathrm{psu}$, which is twice smaller than that predicted by the Visbeck et al.'s (1996) simplified model. From Fig. 15 it can be seen that, near the end of the calculation, the salinity loss exceeds supply for the basic case so that the mean salinity of the polynya slightly decreases. For the other cases, the salinity loss remains smaller than its supply over the duration of the calculation. The limitation on the calculation time required to avoid boundary effects makes it difficult to rigorously interpret this difference between our simulations and the model of Visbeck et al. However, the overshooting of salinity export over import in the basic case polynya may signify that, when a wall is present, attaining equilibrium is not a monotonic process or that the equilibrium stratification of the ambient fluid determined by eddies necessary to balance the buoyancy fluxes, assumed by Visbeck et al., cannot be attained. The increase in the salinity loss cannot be attributed to the coastal current transport: salt volume in the current increases gradually and, to the end of the basic run, constitutes around $10 \%$ of the whole salt volume transported outside the polynya ( $7 \%$ for the double salinity flux case). The fraction of salt contained in the current is much larger if only salt transport west of the polynya is considered: it decreases gradually from $92 \%$ to $62 \%$ during the basic run (to $70 \%$ for the double salinity flux case).

\section{The effect of coastal and bottom topography on the coastal current}

In this section we describe the effect that coastal and basal topography can have on the evolution of a coastal current.

\section{a. Coastal protrusion}

Flow of a current around a cape is studied as the most likely location for the production of anticyclonic lenses of anomalous characteristics in the ocean. The most prominent example of such lenses are the so called meddies shed from the Mediterranean Undercurrent (e.g., Swallow 1969). D'Asaro (1988) proposed that coastal drag gives rise to an anticyclonic layer that, when the current overshoots the cape, produces an eddy. Pichevin and Nof (1995) integrated the steady and inviscid momentum equation around a cape to show that this leads to an unbalanced flow force that should be balanced by shedding eddies off cape. Cenedese and Whitehead (2000) studied experimentally a 

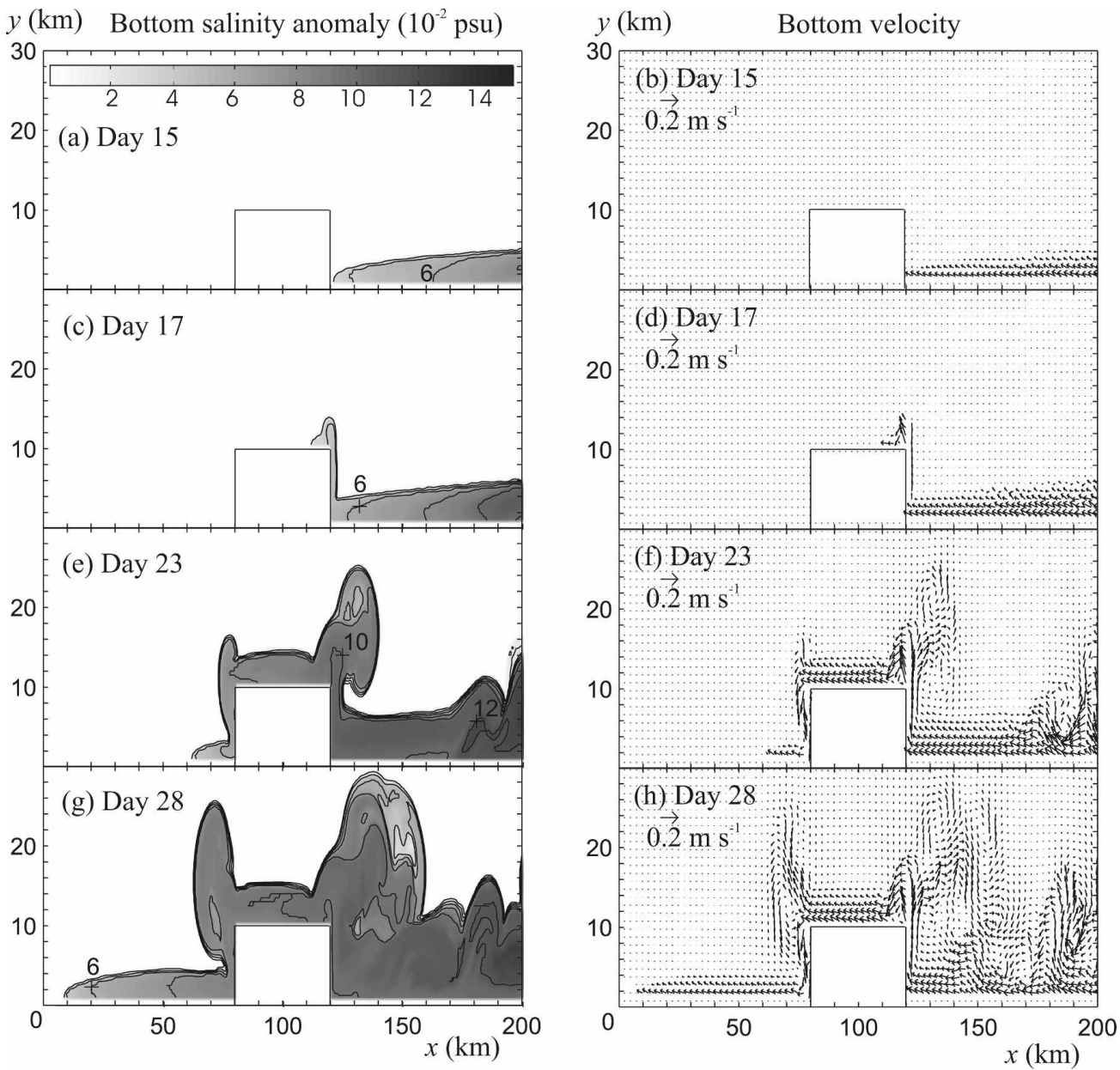

FIG. 16. The bottom (left) salinity anomaly and (right) velocity for the coastal current moving around a coastal protrusion $40 \mathrm{~km}$ long and $40 \mathrm{~km}$ wide positioned around $x=100 \mathrm{~km}$ : all other parameters are as in the basic case.

buoyant jet flowing northward and running past a thin wall protrusion that represented a cape; they found that no eddy shedding was observed for small bottom slopes $(<0.25)$ and eddy formation was inhibited at low current flow rates. Sadoux et al. (2000) performed an experimental study of a current between two layers of different density in a rotating tank and found that no eddies were shed from the cape when the upstream current was stable (at high Rossby numbers). As the Rossby number decreased and the current itself became unstable, the cape became a "privileged place" for lens formation in the form of an anticyclonic eddy or a dipole. The dipole structure was replicated in numerical simulations of a dense current on a sloping bottom by Aiki and Yamagata (2004). Here we only briefly show the basic features of the polynya-generated coastal current on a horizontal bottom that encounters a rectangular protrusion.
We introduced a coastal protrusion of rectangular shape, $40 \mathrm{~km}$ long and $10 \mathrm{~km}$ wide, positioned around $x=100 \mathrm{~km}$ : All other parameters are taken as in the basic case. In Fig. 16 the bottom salinity and velocity in the region surrounding the protrusion are shown after the current approaches it from the east. As the current runs into the protrusion, it turns around the interior, concave corner in a stable fashion. At the next, northeastern, convex corner the wall does not oppose the flow and the current overshoots inertially before the action of the Coriolis force returns the current to move along the wall. This is similar to the experimental observations of Sadoux et al. (2000). By day 18 an anticyclonic eddy attached to the convex corner is formed, accompanied by cyclonic motion southeast of it. By day 22 the anticyclone detaches itself from the corner. The salinity distribution on day 23 at $200-\mathrm{m}$ depth shows that the lens of dense water is about $200 \mathrm{~m}$ deep and its 

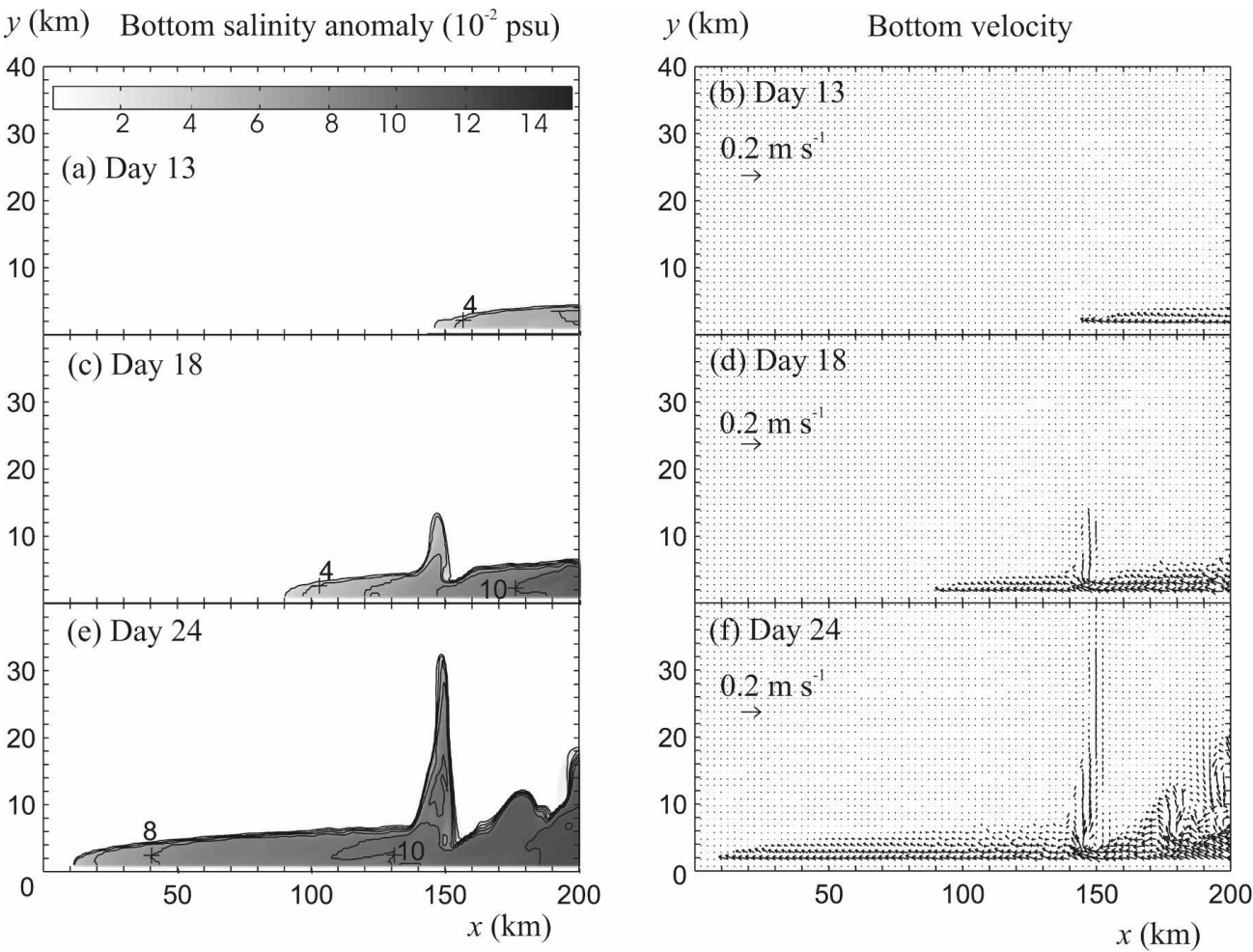

FIG. 17. Same as in Fig. 16, but for the coastal current moving over a 100-m-deep and 10-km-wide canyon positioned at $x=150 \mathrm{~km}$.

peak is around $x=130 \mathrm{~km}$ and $y=15 \mathrm{~km}$, which is between the cyclone and anticyclone of the dipole. Similar to the results of Aiki and Yamagata (2004), we notice that, while the anticyclone subsides at the surface, the cyclone does not.

A similar effect of overshooting and eddy formation occurs at the next, northwest, convex corner where the process is somewhat weaker as part of the kinetic energy of the flow has been transferred into the energy of the eddies off the northeastern corner. The impression that the current is diverted offshore is due to the different scaling of the axes. The current then runs southward into the coast, turns around the interior, concave corner in a stable way and continues westeard. From this simulation, we may infer that, when the bottom coastal current generated by a polynya encounters a cape over a flat bottom, dipole eddies can be shed. This is in contrast to the buoyant current experiments by Cenedese and Whitehead (2000), who showed that no detachment occurred when the topographic $\beta$-plane effect is small. Although Sadoux et al. (2000), in their study of a current between two layers of different density, found that an anticyclone can be shed over a flat bottom, this did not occur when the upstream flow is stable. In our calculations, an eddy detached itself even though the current was stable upstream. The nature of the stability, however, is different: in Sadoux et al. the stability is induced at a large Rossby number, that is, by stronger advection relative to rotation, whereas in our calculations the stability is due to frictional dissipation supressing eddy formation. The potential vorticity gradient of our current changes sign, a necessary condition for instability, so that in the absence of friction instability would be expected upstream. It is worth noting that Aiki and Yamagata (2004) showed that dipole shedding may not be determined by the upstream current instability at all. To understand these differences, more detailed studies would be required, which, however, are out of the scope of this work.

\section{b. Basal canyon}

In this study, we considered the effect of a basal canyon $100 \mathrm{~m}$ deep and $10 \mathrm{~km}$ wide, positioned perpendicular to the coast along $x=150 \mathrm{~km}$; all other parameters are taken as in the basic case. Similar to Chapman and Gawarkiewicz (1995), the canyon shape $D(x)$ is taken to be Gaussian:

$$
D=D_{0} e^{-\left(x-x_{0}\right)^{2} / b^{2}},
$$

where $x_{0}=150 \mathrm{~km}, D_{0}=100 \mathrm{~m}$, and $b=5 \mathrm{~km}$. The bottom salinity and velocities are given in Fig. 17. The 
presence of the canyon does not cause any instability in the motion of the current. The eddies that can be seen at day 24 to the right of $x=150 \mathrm{~km}$ (Fig. 14e) are analogs of those seen in Fig. $2 \mathrm{~g}$ for the basic case. Although part of the current mass is drained into the canyon, there was no significant stagnation of the westward flowing coastal current, as this reached the end of the domain at day 24 as in the basic case.

\section{Concluding remarks}

We used the three-dimensional, hydrostatic ocean model POLCOMS to investigate the ocean flow generated by the surface salt flux in a rectangular Antarctic latent heat polynya. The model geometry used here is particularly relevant to the East Antarctic coastal region. In common with previous calculations, our model reveals a plume of eddies moving offshore; however, our model also revealed a coastal current moving westward along the coast. This coastal current is a robust feature, appearing in simulations addressing a wide range of typical conditions; our calculations suggest the reason a coastal current was not simulated by previous authors (Gawarkiewicz and Chapman 1995) is that it emerges only once a relatively fine horizontal resolution is adopted. As salt is released into the surface waters of the polynya, it first sinks and mixes, densifying a vertical water column beneath the polynya. Cyclonic eddies are then generated at the polynya edge due to vortex tube stretching caused by baroclinic instability. These eddies are superimposed onto an anticyclonic flow due to geostrophic adjustment. As this anticyclonic flow runs into the coast (or landfast ice), a coastal current is generated that moves westward along the coast. The coastal current transports dense, salty water to the western side of the plume and, in our simulation, the westward salinity transport in the current constitutes more than $60 \%$ of the whole westward salinity transport. When the polynya is directly adjacent to the coast, the coastal current is stable in the frontal region and unstable upstream where the current is deep, which concurs with experimental observations (e.g., Condie and Ivey 1988). The upstream barotropic and baroclinic instabilities are opposed by Ekman drainage dissipating the energy of the perturbations. When the polynya is offshore, the coastal current contains eddies from its outset, which is caused by capturing cyclonic eddies at the polynya's southwest corner. Where the coastal current is stable within $50 \mathrm{~km}$ of its front, its mean speed, normalized by the mean gravity-inertia wave speed calculated using local densities and depths over the $50 \mathrm{~km}$ of the current's length, is in the range $0.4 \pm 0.2$ : the current width, normalized by the mean local Rossby radius, is in the range of $1.9 \pm 0.3$. When a rectangular coastal protrusion is present, the current flows around the protrusion (from east to west), flowing smoothly around interior, concave corners but overshooting inertially at exterior, convex corners. In the latter case, a dipole lens is shed from the current. The presence of a 100-m deep canyon splits the current in two, but does not introduce any noticeable instability.

Acknowledgments. Insightful remarks of two anonymous reviewers helped us to improve the paper. The authors are grateful to Andrew Willmott, James Holt, Clare Postlethwaite, and Graham Tattersall of the Proudman Oceanographic Laboratory for consultations and technical support regarding the POLCOMS model, and we are grateful for the use of the UCL Research Computing Keter cluster.

\section{REFERENCES}

Aiki, H., and T. Yamagata, 2004: A numerical study on the successive formation of Meddy-like lenses. J. Geophys. Res., 109, C06020, doi:10.1029/2003JC001952.

Bacon, S., G. Reverdin, I. G. Rigor, and H. Snaith, 2002: A freshwater jet on the East Greenland shelf. J. Geophys. Res., 107, 3068, doi:10.1029/2001JC000935.

Baines, P. G., and S. Condie, 1998: Observations and Modelling of Antarctic Downslope Flows: A Review. AGU Antarctic Research Series, Vol. 75, Amer. Geophys. Union, 29-49.

Brickman, D., 1995: Heat flux partitioning in open-ocean convection. J. Phys. Oceanogr., 25, 2609-2623.

Cavalieri, D. J., and S. Martin, 1985: A Passive Microwave Study of Polynyas along the Antarctic Wilkes Land Coast. AGU Antarctic Research Series, Vol. 43, Amer. Geophys. Union, 227-252.

Cenedese, C., and J. A. Whitehead, 2000: Eddy shedding from a boundary current around a cape over a sloping bottom. $J$. Phys. Oceanogr., 30, 1514-1531.

Chapman, D. C., and G. Gawarkiewicz, 1995: Offshore transport of dense shelf water in the presence of a submarine canyon. J. Geophys. Res., 100, 13 373-13 387.

Condie, S. A., and G. N. Ivey, 1988: Convectively driven coastal currents in a rotating basin. J. Mar. Res., 46, 473-494.

D'Asaro, E., 1988: Generation of submesoscale vortices: A new mechanism. J. Geophys. Res., 93, 6685-6693.

Davies, P. A., and I. Ahmed, 1996: Laboratory studies of a round, negatively buoyant jet discharged horizontally into a rotating homogeneous fluid. Fluid Dyn. Res., 17, 237-274.

_, H. J. S. Fernando, F. Besley, and R. J. Simpson, 1991: Generation and spreading of a turbulent mixed layer in a rotating, stratified fluid. J. Geophys. Res., 96, 12 567-12 585.

Deleersnijder, E., and P. Luyten, 1994: On the practical advantages of the quasi-equilibrium version of the Melllor and Yamada level 2.5 turbulence closure applied to numerical modelling. Appl. Math. Modell., 18, 281-287.

Dewar, W. K., and P. D. Killworth, 1990: On the cylinder collapse problem, mixing, and merger of isolated eddies. J. Phys. Oceanogr., 20, 1563-1575.

Etling, D., F. Gelhardt, U. Schrader, F. Brennecke, G. Kühn, C. d'Hieres, and H. Didelle, 2000: Experiments with density cur- 
rents on a sloping bottom in a rotating fluid. Dyn. Atmos. Oceans, 31, 139-164.

Ezer, T., 2005: Entrainment, diapycnical mixing and transport in three-dimensional bottom gravity current simulations using the Mellor-Yamada turbulence scheme. Ocean Modell., 9, 151-168.

Galperin, B., L. H. Kantha, S. Hassid, and A. Rossati, 1988: A quasi-equilibrium turbulent energy model for geophysical flows. J. Atmos. Sci., 45, 55-66.

Gawarkiewicz, G., 2000: Effect of ambient stratification and shelfbreak topography on offshore transport of dense water on continental shelves. J. Geophys. Res., 105, 3307-3324.

— and D. C. Chapman, 1995: A numerical study of dense water formation and transport on a shallow, sloping continental shelf. J. Geophys. Res., 100, 4489-4507.

Griffiths, R. W., and P. F. Linden, 1982: Laboratory experiments on fronts. Part I: Density-driven boundary currents. Geophys. Astrophys. Fluid Dyn., 19, 159-187.

—, 1986: Gravity currents in rotating systems. Annu. Rev. Fluid Mech., 18, 59-89.

- , and P. F. Linden, 1981: The stability of buoyancy-driven coastal currents. Dyn. Atmos. Oceans, 5, 281-306.

- , P. D. Killworth, and M. E. Stern, 1982: Ageostrophic instability of ocean currents. J. Fluid Mech., 117, 343-377.

Grumbine, R. W., 1991: A model of the formation of high-salinity shelf water on polar continental shelves. J. Geophys. Res., 96, 22 049-22 062.

Holt, J. T., and I. D. James, 1999: A simulation of the southern North Sea in comparison with measurements from the North Sea Project. Part 1: Temperature. Cont. Shelf Res., 19, 10871112.

, and - 2001: An $s$ coordinate density evolving model of the northwest European continental shelf. 1. Model description and density structure. J. Geophys. Res., 106, 14 01514034.

Ikeda, M., 1987: Wind effects on the buoyancy-driven general circulation in a closed basin using a two-level model. J. Phys. Oceanogr., 17, 1707-1723.

Ivanov, V. V., G. I. Shapiro, J. M. Huthnance, D. L. Aleynik, and P. N. Golovin, 2004: Cascades of dense water around the world ocean. Prog. Oceanogr., 60, 47-98.

Ivey, J., J. Taylor, and M. Coates, 1995: Convectively driven mixed layer growth in a rotating, stratified fluid. Deep-Sea Res. I, 42, 331-349.

James, I. D., 1996: Advection schemes for shelf sea models. $J$. Mar. Syst., 8, 237-254.

Jiang, L., and R. W. Garwood Jr., 1996: Three-dimensional simulation of overflows on continental slopes. J. Phys. Oceanogr., 26, 1214-1233.

Johannessen, O. M., and M. Mork, 1979: Remote sensing experiment in the Norwegian coastal waters. Geophysisk Institutt, Universitetet i Bergen Tech. Rep. 3/79.

Killworth, P. D., 1985: A two-level wind and buoyancy driven thermocline model. J. Phys. Oceanogr., 15, 1414-1432.

Kukichi, T., M. Wakatachi, and M. Ikeda, 1999: A numerical investigation of the transport process of dense shelf water from a continental shelf to slope. J. Geophys. Res., 104, 1197-1210.

Lane-Serff, G. F., and P. G. Baines, 1998: Eddy formation by dense flows on slopes in a rotating fluid. J. Fluid Mech., $\mathbf{3 6 3}$, 229-252.

Legg, S., and J. Marshall, 1993: A heton model of the spreading phase of open-ocean deep convection. J. Phys. Oceanogr., 23, 1040-1056.

— , R. W. Hallberg, and J. B. Girton, 2006: Comparison of entrainment in overflows simulated by $z$-coordinate, isopycnal and nonhydrostatic models. Ocean Modell., 1, 69-97.

Massom, R. A., P. T. Harris, K. J. Michael, and M. J. Potter, 1998: The distribution and formative processes of latent-heat polynyas in east Antarctica. Ann. Glaciol., 27, 420-426.

Mellor, G. L., 1989: Retrospective on oceanic boundary layer modelling and second moment closure. The Parameterisation of Small Scale Processes: Proc. 'Aha Huliko'a Hawaiian Winter Workshop, Honolulu, HI, University of Hawaii at Manoa, 251-271.

1991: An equation of state for numerical models of oceans and estuaries. J. Atmos. Oceanic Technol., 8, 601-611.

, and T. Yamada, 1974: A hierarchy of turbulence closure models for planetary boundary layers. J. Atmos. Sci., 31, 1791-1806.

Morales Maqueda, M. A., A. J. Wilmott, and N. R. T. Biggs, 2004: Polynya dynamics: A review of observations and modeling. Rev. Geophys., 42, RG1004, doi:10.1029/2002RG000116.

Nof, D., 1988a: Draining vortices. Geophys. Astrophys. Fluid Dyn., 42, 187-208.

— 1988b: Eddy-wall interactions. J. Mar. Res., 46, 527-555.

Pichevin, T., and D. Nof, 1995: The eddy cannon. Deep-Sea Res. I, 43, 1475-1507.

Rintoul, S. R., J. R. Donguy, and D. H. Roemmich, 1997: Seasonal evolution of upper ocean thermal structure between Tasmania and Antarctica. Deep-Sea Res. I, 44, 1185-1202.

Sadoux, S., J. Baey, A. Fincham, and D. Renouard, 2000: Experimental study of the stubility of an intermediate current and its interaction with a cape. Dyn. Atmos. Oceans, 31, 165-192.

Sansón, L. Z., F. Greaf, and E. G. Pavía, 1998: Collision of anticyclonic, lens-like eddies with a meridional western boundary. J. Geophys. Res., 103, 24 881-24 890.

Shi, C., and D. Nof, 1994: The destruction of lenses and generation of wodons. J. Phys. Oceanogr., 24, 1120-1136.

Smith, S. D., R. D. Muench, and C. H. Pease, 1990: Polynyas and leads: An overview of physical processes and environment. $J$. Geophys. Res., 95, 9461-9479.

Swallow, J. C., 1969: A deep eddy off Cape St. Vincent. Deep-Sea Res., 16, 285-295.

Swaters, G. E., 1991: On the baroclinic instability of cold-core coupled density fronts on a sloping continental shelf. J. Fluid Mech., 224, 361-382.

Tanaka, K., and K. Akimoto, 2001: Baroclinic instability of density current along a sloping bottom and the associated transport process. J. Geophys. Res., 106, 2621-2638.

Visbeck, M., J. Marshall, and H. Jones, 1996: Dynamics of isolated convective regions in the ocean. J. Phys. Oceanogr., 26, 17211734.

Wadhams, P., A. Gill, and P. F. Linden, 1979: Transects by submarine of the East Greenland Polar Front. Deep-Sea Res., 26, 1311-1327. 